\title{
Allanite-britholite bearing migmatite gneiss-Amet Granite and intrusive syenite-monzonite rocks of SW Rajasthan, India, and their REE potential
}

\author{
${ }^{1}$ Dy. Director General (Retd.), Geological Survey of India; Residence: B-230, 10B Scheme, Gopalpura Bypass Road, Jaipur, India, PIN Code- \\ 302018; *Corresponding author, E-mail:dbguha@gmail.com \\ ${ }^{2}$ Senior Geologist, Geological Survey of India, 15-16, Jhalana Dungri, Jaipur, India, PIN Code-302004 \\ ${ }^{3}$ Senior Geologist, Geological Survey of India, SU-WB\&AN, Salt Lake, Kolkata, India, PIN Code-700091 \\ ${ }^{4}$ Senior Geologist, NCEGR, Geological Survey of India, Bengaluru, India, PIN Code-560070
}

(Received: July 8, 2018; Revised accepted: November 15, 2019)

https://doi.org/10.18814/epiiugs/2020/020044

\begin{abstract}
Allanite and REE bearing minerals are primum fama report from migmatite gneisses, plutonic Amet granite and intrusive alkaline rocks of Rajasthan, and these minerals are important for establishing REE deposit. Allanite taken from host migmatite and granite have been analysed along with host rocks containing REE minerals, both of which contain appreciably high amount of $\Sigma R E E$ and Th. Allanite phases identified are $\mathrm{CaO}$ and $\mathrm{Ce}_{2} \mathrm{O}_{3}$ rich with positive correlation of silica and alumina. As silica proportion decreases and $\Sigma R E E$ increases, allanite changes to britholite and thorite indicating a role of fluid in its crystallization. The chondrite normalized REE in migmatite gneiss and allanite mineral is compatible indicating a primary nature of allanite. The carrier of REE minerals is envisaged as the alkaline intrusives which deposited their REE minerals during ascent of magma from deeper source. Chemico-mineralogical study of Allanite and its hosts suggest possible reaction between a REE bearing fluid rich in $P$ and $F$ brought up by alkaline rocks, and the silicate minerals, mainly biotite and hornblende of the migmatite gneiss and Amet granite. The allanite in migmatite gneiss and granite may prove to be a sumptuous deposit of REE minerals in India, and similar terrains elsewhere.
\end{abstract}

\section{Introduction}

The NW Indian craton of Rajasthan, comprising northwestern edge of the Indian shield consists predominantly of gneiss-granite-amphibolite-metasedimentary rock association of Archaean age, referred to as the Banded Gneissic Complex or BGC (Gupta, 1934; Heron, 1953; Raja Rao, 1970). The gneissic complex served as basement over which the Proterozoic cover rocks of Aravalli and Delhi Supergroup, along with the platformal sediments of Vindhyan Supergroup, were deposited with profound unconformities (Heron, op.cit.; Raja Rao et al., 1971; Prasad, 1984; Sinha Roy, 2000). The BGC basement has stabilized at about $2500 \mathrm{Ma}$ (Wiedenbeck et al., 1996).

During Archaean-Proterozoic times, the Rajasthan craton witnessed formation of greenstone sequences and granulite gneisses (Mohanty and Guha, 1995; Guha, 2018). The deformational and metamorphic episodes led to crystallisation of migmatite gneisses with several episodes of anatectic melting, and emplacement of several intrusive magmatic plutons (Guha et al., 2018). The greenstone sequences are reworked in later deformations and are a storehouse of several known $\mathrm{Pb}-\mathrm{Zn}-\mathrm{Cu}-\mathrm{Au}-\mathrm{U}$ deposits in India, e.g., Kayar-Ghugra $\mathrm{Pb}-\mathrm{Zn}$ deposit, Pur Banera Pb-Zn-Cu deposit (Oza et al., 2017), Agucha Zn-Pb deposit (Gandhi, 2003; Fareeduddin et al., 2014), Rohil (Khetri fold belt) U deposit (Nanda et al., 2009), Rare metal and REE deposit in BGC terrain of Bichun-Nayagaon (Shaji et al., 2011; Yadav et al., 2015). Occurrence of rare minerals, such as, Ecandrewsite has recently been reported from the Khetri fold belt of Rajasthan having its significance in REE and $\mathrm{Au}-\mathrm{Cu}-\mathrm{U}$ search (Neogi et al., 2017).

During course of our investigations on Archaean terrane of SW Rajasthan, India, we discovered primum fama widespread occurrence of allanite, which is one of the major REE bearing mineral, around Borana, Mad, Rama and Tikar areas of Rajsamand district of Rajasthan State, India (Fig. 1). In view of present worldwide demands of REE, the detail study of REE bearing minerals is highly required. This new find of REE bearing minerals in migmatite in recoverable resources will be a new emerging domain in India. It was, therefore, decided to study the allanite composition to know the concentration of different REE within it and also to compare the allanite composition of present finding with the composition of allanite commonly occurring worldwide. Allanite $(\mathrm{Ca}, \mathrm{REE})_{2}\left(\mathrm{Fe}, \mathrm{Al}_{3} \mathrm{Si}_{3} \mathrm{O}_{12}[\mathrm{OH}]\right.$, is a REE enriched mineral of epidote group, is being first time reported from the migmatite gneisses (Fig. 1b) and their associate alkaline intrusive bodies of Syenite/alkali feldspar syenite (Fig. 1a), and granite, referred to as Amet Granite, in the study area. REE deposits of the world are mainly found in carbonatite, as in Africa; Fe-carbonate rocks, or clays as in China deposits (Harmer and Nex, 2016). In Bayan Obo, Inner Mongolia, REE ore in silicate rock is found and the economically exploited minerals are bastnaesite and monazite. Other considerable occurrences of rare earth 

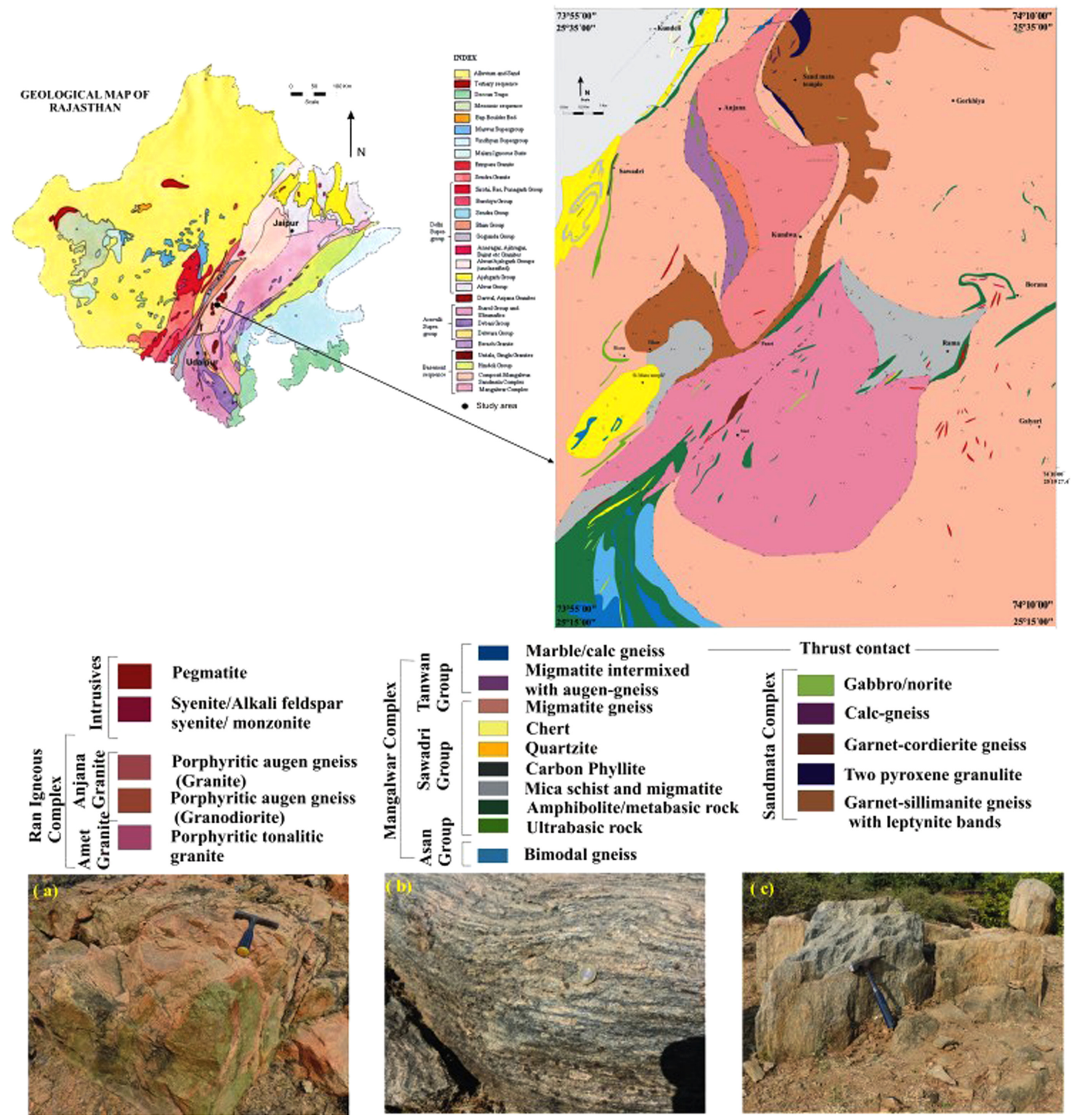

Figure 1. Geological map of Borana-Mad area showing disposition of lithologies, (a) Syenite/Alkali feldspar syenite exposed at Chatrawan mata temple near Borana, (b) Migmatite gneiss outcrop with dispersed allanite grains (brown spots) with haloes, (c) Intrusive monzonite rock within the Amet granite near Mad. Action of fluid is noticeable as foliation parallel replacement of Amet granite (grey) by monzonite (pink).

mineral resources are related to alkaline igneous intrusive complexes and carbonatite intrusive in Paleo Proterozoic orthogneiss and granite gneiss, such as Mountain Pass wherein the main ore minerals are bastnaesite, allanite, monazite and apatite (Olson et al., 1951; Woyski and Margaret, 1980). Nowhere throughout the world, is occurrence of REE mineralisation in migmatite gneisses reported, as discussed above.

The profuse occurrence of allanite in the migmatite gneisses, Amet Granite pluton, and syenite/alkali feldspar syenite of Borana and Mad areas (Fig. 1) of present study is of significance, as it has not been reported so far from the cratonic terrane (Basement gneisses of BSG) of Rajasthan, India or rarely elsewhere in the world also. The migmatite gneiss of study area covers almost fifty percent and is exposed in the east as well as in the western parts around Borana, Mad, Rama and north and south of Tikar (Fig. 1). The Amet Granite pluton is oval in shape, covering about 30 per cent of the area, having its western boundary incised by a longitudinal shear zone running northeasterly (Fig. 1).

Occurrence of REE bearing allanite with its alteration to britholite, thorite along with zircon, titanite, apatite in basement rock migmatite 
gneiss (silicate rock) intruded by syenite (alkaline rock), and plutonic Amet Granite in cratonic part of Rajasthan, is unique in its rarity. This new find of allanite, in recoverable profuse quantity, may turn up into a mineable deposit of REE in widespread migmatite gneiss-granite complexes of Rajasthan and elsewhere in similar cratonic terranes of India and the world. In present study, authors tried to find out the behaviour of different rare earth elements within allanite and its host rock; thereby highlighting its origin during possible magmatic crystallisation. The chondrite normalised pattern of $\Sigma$ REE in allanite vis-a-vis the chondrite normalised pattern of $\Sigma$ REE in rocks containing these allanite are compared and a compatible relation of $\Sigma$ REE of rock visa-vis the mineral allanite contained within it is observed; indicating allanite of primary magmatic origin (Fig. 20, see also at p.15 line 373-376). Since allanite crystallised in later period of magmatic crystallisation, the compositional variation in allanite REE and host REE is imminent.

Aim of the paper is to establish behaviour of REE in allanite, and the relation between allanite and the host rocks through detailed chemicomineralogical study of both REE bearing allanite and allanite bearing host rocks, leading to establishment of recoverable REE deposit in migmatite cratonic terrain of Rajasthan, India.

\section{Geological Setting}

The NW Indian craton of Rajasthan (also known as Rajasthan craton or Aravalli craton), made up mostly of the Banded Gneissic Complex (BGC), is flanked on the west by the Proterozoic fold belts of the Aravalli and Delhi Supergroup rocks (Heron, 1953) that has attracted the attention of scientists because of its heterogeneous lithological assemblages, tectonic stacking, and early Archaean to mid Proterozoic complex magmatic-metamorphic history. The BGC has been reclassified as the Bhilwara Supergroup (BSG, Raja Rao, 1970; Gupta et al., 1997) which is made up of lithologies of granulite facies Sandmata Complex (SC, 2.29-1.72 Ga; Sarkar et al., 1989; Buick et al., 2006), amphibolite facies Mangalwar Complex (MC, 3.3-2.8 Ga; Gopalan et al., 1990) and greenschist facies Hindoli Group (younger than 2.52 Ga; Dey et al., 2018). The geology of the BSG has been worked out in detail by Guha and Bhattacharya (1995), Mohanty and Guha (1995), Gupta et al. (1997), Chakrabarti et al. (2004), Guha (2018), and specially the geological and petrological details of the migmatite gneisses and its enclosing granite pluton (Anjana Granite) north of the present granite pluton (Amet Granite) is dealt in detail by Guha et al. (2018).

\section{Rock Types Containing Allanite}

Migmatite gneiss (protolith age 2.75-2.698 Ga, Dharma Rao et al., 2011 ) is a mixed rock and consists of mainly two parts, leucosome and palaeosome. Leucosome consists of mainly quartz and feldspar (K-feldspar and plagioclase), where as paleosome consists of biotite, amphibole \pm pyroxene \pm garnet. Large body of amphibolite occurs within migmatite gneiss with diffuse boundary near Mad, Rama and Borana areas (Fig. 1). Dispersed grains of primary allanite with light brown alteration rims are found in migmatite gneisses around BoranaRama-Galyari areas (Fig. 1b). The $1-2 \mathrm{~mm}$ to $10 \mathrm{~mm}$ size grains of allanite occur along the foliation defined by biotite-muscovite of migmatite gneiss, lying either along the foliation or athwart to it, and many times pushing across the foliation. The light brown alteration rims around these allanite grains are 1 to $3 \mathrm{~mm}$ in thickness.

Amet Granite (1870 \pm 200 Ma, Chaudhary et al., 1984) covers large part of the mapped area around Mad (Fig. 1). The Amet Granite is intrusive within the migmatite rock and is considered a product of partial melting of migmatite gneisses of the Mangalwar Complex (Guha, 2018; Guha et al., 2018). This plutonic body is semi-circular with moderately developed foliation in the core part. The peripheral part of the Amet Granite is sheared with development of mylonite zone where garnet and the feldspar phenocrysts are extremely sheared. The western part of this granite body is incised by a longitudinal northeasterly trending major shear zone (Fig. 1). The Amet Granite is medium to coarse grained and is tonalitic in composition, comprising of plagioclase, quartz with small amount of alkali feldspars and hornblende and subordinate amount of biotite. Primary, discrete and euhedral crystals of allanite are seen within this granite.

Amphibolite (2.83 $\pm 0.05 \mathrm{Ga}$, Gopalan et al., 1990) occurs as bands of different size and shape within the Amet granite and migmatite gneiss. Some of the amphibolite bands are large enough to be mapped in the present scale. Near Borana and Rama, thick amphibolite bands are exposed which are highly deformed and show multiple folding (Fig. 1). It is a medium grain, dark colour rock consisting of amphibole as dominant mineral with feldspar and minor biotite, apatite, sphene, opaque minerals.

At Chatrawan mata temple, near Borana, younger intrusive plutonic bodies identified as syenite-alkali feldspar syenite (Fig. 1a) are found associated with amphibolite. Nevertheless, these syenite-alkali feldspar syenite are intrusive within the host migmatite gneiss in which amphibolite has intruded migmatite gneiss syntectonically and is folded along with it (Fig. 1). These younger intrusive alkaline plutonic bodies are nonfoliated and extend in the east-west direction which are also marked with numerous criss-cross epidote veins of varying size having dispersed allanite grains. These allanite grains are dark in colour with conchoidal fractures and light brown haloes around it.

Small unmappable intrusive plutonic body of alkali feldspar rich rock monzonite was identified as intrusive body within the Amet Granite near west of Mad. This monzonite is extending in the eastwest direction showing intrusive nature with the Amet Granite (Fig. 1c). The monzonite and syenite-alkali feldspar syenite are hitherto not reported from this BSG terrain. Therefore, it is clear from field interrelations that the syenite-alkali feldspar syenite and the monzonite are intrusive both within the migmatite gneiss and the Amet Granite respectively and its relation with amphibolite is inferred. As amphibolite is syntectonically emplaced within migmatite gneiss and is folded with migmatite, whereas the alkaline rocks are undeformed, it can be said that the alkaline rocks are the latest in its emplacement in the area. Isotopic ages of alkaline rocks are not yet determined.

\section{Dataset and Sample Collection}

Samples were collected from the different part as well as different phases of the migmatite gneiss, granites and also from the associated late acidic intrusions to examine mineralogical and chemical variations within the same pluton and also between different granitic phases. Fresh samples containing megascopically visible allanite grains in rock were 
Table 1. Whole rock and trace element chemical analysis data of Amet Granite

\begin{tabular}{|c|c|c|c|c|c|c|c|c|}
\hline \multirow[b]{2}{*}{ Sample No. } & \multicolumn{8}{|c|}{ Amet Granite } \\
\hline & PCS- 01/08 & PCS- 06/08 & PCS- 22/08 & PCS- 23/08 & PCS- $24 / 08$ & PCS- $25 / 08$ & PCS- 26/08 & PCS- $27 / 08$ \\
\hline $\mathrm{SiO}_{2}$ & 69.49 & 60.1 & 61.14 & 59.46 & 58.9 & 61.23 & 58.89 & 57.19 \\
\hline $\mathrm{Al}_{2} \mathrm{O}_{3}$ & 13.86 & 14.12 & 13.56 & 13.74 & 13.55 & 13.14 & 13.52 & 13.37 \\
\hline $\mathrm{Fe}_{2} \mathrm{O}_{3}$ & 1.86 & 6.2 & 6.45 & 6.37 & 7.08 & 6.26 & 7.44 & 8.62 \\
\hline $\mathrm{MnO}$ & 0.02 & 0.04 & 0.07 & 0.08 & 0.09 & 0.07 & 0.08 & 0.1 \\
\hline $\mathrm{MgO}$ & 0.13 & 2.48 & 1.19 & 1.7 & 1.47 & 1.23 & 2.18 & 1.68 \\
\hline $\mathrm{CaO}$ & 1.19 & 4.31 & 2.65 & 3.26 & 3.84 & 2.79 & 3.73 & 3.78 \\
\hline $\mathrm{Na}_{2} \mathrm{O}$ & 2.58 & 2 & 1.83 & 1.93 & 2.14 & 1.94 & 1.87 & 2.3 \\
\hline $\mathrm{K}_{2} \mathrm{O}$ & 5.71 & 2.62 & 5.99 & 5.62 & 4.79 & 5.8 & 4.53 & 4.97 \\
\hline $\mathrm{TiO}_{2}$ & 0.03 & 1.18 & 0.88 & 0.85 & 1.05 & 0.87 & 1.2 & 1.31 \\
\hline $\mathrm{P}_{2} \mathrm{O}_{5}$ & 0.01 & 0.02 & 0.34 & 0.37 & 0.48 & 0.35 & 0.56 & 0.56 \\
\hline $\mathrm{Ba}$ & 55 & 1170 & 1860 & 1344 & 1503 & 1486 & 1241 & 1925 \\
\hline Co & 7 & 31 & 27 & 27 & 30 & 28 & 27 & 26 \\
\hline $\mathrm{Cr}$ & $<15$ & 58 & $<15$ & 28 & $<15$ & 16 & 45 & $<15$ \\
\hline $\mathrm{Cu}$ & $<1$ & 8 & 10 & 12 & 10 & 12 & 20 & 16 \\
\hline $\mathrm{Ga}$ & 25 & 23 & 18 & 18 & 18 & 18 & 21 & 23 \\
\hline $\mathrm{Nb}$ & 56 & 15 & 33 & 25 & 26 & 32 & 34 & 62 \\
\hline $\mathrm{Pb}$ & 44 & 9 & 19 & 23 & 19 & 31 & 25 & 16 \\
\hline $\mathrm{Rb}$ & 455 & 93 & 188 & 168 & 120 & 171 & 133 & 180 \\
\hline $\mathrm{Sc}$ & $<3.5$ & 10 & 10 & 7 & 9 & 7 & 13 & 19 \\
\hline $\mathrm{Sr}$ & 17 & 260 & 243 & 216 & 267 & 213 & 242 & 267 \\
\hline Th & 59 & $<4$ & 11 & 7 & 8 & 15 & $<4$ & 24 \\
\hline $\mathrm{V}$ & $<20$ & 222 & 60 & 72 & 81 & 63 & 113 & 75 \\
\hline $\mathrm{Y}$ & 260 & $<5$ & 39 & 41 & 43 & 45 & 49 & 57 \\
\hline $\mathrm{Zn}$ & 11 & 48 & 63 & 59 & 64 & 67 & 87 & 107 \\
\hline $\mathrm{Zr}$ & 142 & 357 & 427 & 287 & 357 & 413 & 333 & 674 \\
\hline $\mathrm{Ni}$ & 6 & 31 & 10 & 15 & 7 & 11 & 17 & 3 \\
\hline $\mathrm{Be}$ & 1.73 & 3.21 & 2.19 & 2.28 & 1.88 & 2.41 & 10.46 & 2.79 \\
\hline $\mathrm{Ge}$ & 1.7 & 1.73 & 1.42 & 1.44 & 1.49 & 1.45 & 1.46 & 1.4 \\
\hline $\mathrm{Y}$ & 209.3 & 82.33 & 56.56 & 54.39 & 55.25 & 58.12 & 17.17 & 53.02 \\
\hline Mo & $<0.50$ & 1.03 & 0.98 & 1.04 & 0.7 & 1.13 & $<0.50$ & 0.64 \\
\hline $\mathrm{Sn}$ & 2.88 & 2.2 & 1.13 & 1.24 & 1.2 & 1.01 & 1.01 & 2.31 \\
\hline $\mathrm{La}$ & 57.37 & 297.79 & 121.83 & 126.19 & 103.51 & 154.93 & 34.28 & 189.8 \\
\hline $\mathrm{Ce}$ & 113.9 & 460.4 & 202.4 & 211.7 & 195.9 & 258.6 & 60.7 & 292.4 \\
\hline $\operatorname{Pr}$ & 13.58 & 48.56 & 25.56 & 25.05 & 22.06 & 33.07 & 6.08 & 33.91 \\
\hline $\mathrm{Nd}$ & 56.31 & 167.62 & 99.14 & 95.49 & 87.62 & 126.79 & 22.75 & 118.22 \\
\hline $\mathrm{Sm}$ & 17.23 & 20.44 & 15.04 & 14.03 & 13.43 & 18.78 & 5.27 & 14.84 \\
\hline $\mathrm{Eu}$ & 0.43 & 2.67 & 5.63 & 4.68 & 4.37 & 5.93 & 1.01 & 2.38 \\
\hline $\mathrm{Gd}$ & 13.51 & 22.52 & 12.86 & 12.79 & 11.03 & 15.81 & 4.39 & 14.4 \\
\hline $\mathrm{Tb}$ & 4.78 & 3.52 & 2.4 & 2.24 & 2.15 & 2.67 & 1.09 & 2.4 \\
\hline Dy & 39.22 & 15.53 & 11.18 & 10.45 & 10.57 & 11.29 & 4.81 & 10.42 \\
\hline Ho & 9.31 & 3.22 & 2.31 & 2.19 & 2.19 & 2.27 & 0.65 & 2.13 \\
\hline $\mathrm{Er}$ & 24.84 & 10 & 6.7 & 6.54 & 6.13 & 7.04 & 1.35 & 6.49 \\
\hline $\mathrm{Tm}$ & 4.69 & 1.28 & 0.87 & 0.86 & 0.8 & 0.87 & 0.13 & 0.82 \\
\hline $\mathrm{Yb}$ & 30.74 & 8.6 & 5.92 & 5.64 & 5.36 & 5.88 & 1.29 & 5.59 \\
\hline $\mathrm{Lu}$ & 4.47 & 1.17 & 0.8 & 0.78 & 0.71 & 0.8 & 0.17 & 0.77 \\
\hline $\mathrm{Hf}$ & 10.56 & 14.31 & 15.09 & 16.99 & 12.83 & 22.57 & 10.03 & 12.17 \\
\hline $\mathrm{Ta}$ & 2.37 & 1.82 & 1.51 & 1.6 & 1.38 & 2.13 & 0.86 & 0.99 \\
\hline W & 4.7 & 0.95 & 5.52 & 1.74 & 2.07 & 2.65 & 5.91 & 0.85 \\
\hline $\mathrm{U}$ & 10.84 & 3.53 & 0.74 & 1.2 & $<0.50$ & 1.75 & $<0.50$ & 2.4 \\
\hline
\end{tabular}


Table 2. Whole rock and trace element chemical analysis data of migmatite, syenite and amphibolite

\begin{tabular}{|c|c|c|c|c|c|c|c|c|c|c|c|c|c|c|c|c|c|c|c|c|}
\hline Sample No. & $\mathrm{SiO}_{2}$ & $\mathrm{Al}_{2} \mathrm{O}_{3}$ & $\mathrm{Fe}_{2} \mathrm{O}_{3}$ & $\mathrm{MnO}$ & $\mathrm{MgO}$ & $\mathrm{CaO}$ & $\mathrm{Na}_{2} \mathrm{O}$ & $\mathrm{K}_{2} \mathrm{O}$ & $\mathrm{TiO}_{2}$ & $\mathrm{P}_{2} \mathrm{O}_{5}$ & $\mathrm{Ba}$ & Co & $\mathrm{Cr}$ & $\mathrm{Cu}$ & $\mathrm{Ga}$ & $\mathrm{Nb}$ & $\mathrm{Pb}$ & $\mathrm{Rb}$ & $\mathrm{Sc}$ & $\mathrm{Sr}$ \\
\hline PCS-11/08 & 63.24 & 14.03 & 4.72 & 0.06 & 1.37 & 2.99 & 2.97 & 4.32 & 0.62 & 0.24 & 895 & 22 & 18 & 8 & 19 & 36 & 26 & 201 & 6 & 204 \\
\hline PCS-20/08 & 57.68 & 17.72 & 3.45 & 0.03 & 0.07 & 5.17 & 6.01 & 3.21 & 0.54 & 0.16 & 659 & 24 & 29 & $<1$ & 21 & 71 & 21 & 83 & 5 & 600 \\
\hline BRS-07/08 & 68.58 & 13.36 & 3.46 & 0.04 & 0.45 & 1.44 & 2.24 & 5.67 & 0.33 & 0.08 & 899 & 42 & $<15$ & 2 & 18 & 36 & 34 & 181 & $<3.5$ & 115 \\
\hline BRS-09/08 & 57.18 & 18.4 & 3.75 & 0.03 & 0.02 & 6.29 & 4.37 & 5.52 & 0.45 & 0.21 & 814 & 18 & $<15$ & 6 & 36 & 90 & 36 & 203 & 11 & 847 \\
\hline BRS-10/07 & 59.7 & 18.59 & 3.57 & 0.03 & 0.08 & 5.64 & 7.13 & 2.26 & 0.37 & 0.16 & 585 & 29 & 22 & $<1$ & 28 & 42 & 18 & 47 & 5 & 797 \\
\hline BRS-11/08 & 47.5 & 11.48 & 14.49 & 0.21 & 6.51 & 10.76 & 2.09 & 0.42 & 0.98 & 0.11 & 139 & 57 & 174 & 108 & 22 & 14 & $<2$ & 19 & 48 & 100 \\
\hline BRS-12/07 & 68 & 12.14 & 2.33 & 0.03 & 0.13 & 4.74 & 0.4 & 7.35 & 0.27 & 0.08 & 889 & 29 & $<15$ & 3 & 22 & 65 & 26 & 230 & $<3.5$ & 351 \\
\hline BRS-13/08 & 70.97 & 12.64 & 2.09 & 0.03 & 0.15 & 2.58 & 1.46 & 7.14 & 0.22 & 0.07 & 883 & 38 & $<15$ & 3 & 15 & 48 & 36 & 249 & $<3.5$ & 196 \\
\hline BRS-14/08 & 74.12 & 13.71 & 0.41 & 0.02 & 0.09 & 0.45 & 2.9 & 6.87 & 0.05 & 0.02 & 421 & 34 & $<15$ & $<1$ & 10 & 20 & 25 & 212 & $<3.5$ & 100 \\
\hline BRS-15/08 & 69.34 & 14.26 & 1.65 & 0.02 & 0.32 & 2.26 & 2.4 & 7.05 & 0.27 & 0.08 & 744 & 42 & $<15$ & $<1$ & 17 & 27 & 25 & 229 & $<3.5$ & 176 \\
\hline & Th & $\mathrm{V}$ & $\mathrm{Y}$ & $\mathrm{Zn}$ & $\mathrm{Zr}$ & $\mathrm{Ni}$ & $\mathrm{Be}$ & $\mathrm{Ge}$ & $\mathrm{Y}$ & Mo & $\mathrm{Sn}$ & $\mathrm{La}$ & $\mathrm{Ce}$ & $\operatorname{Pr}$ & $\mathrm{Nd}$ & $\mathrm{Sm}$ & $\mathrm{Eu}$ & $\mathrm{Gd}$ & $\mathrm{Tb}$ & Dy \\
\hline PCS-11/08 & 37 & 36 & 62 & 56 & 376 & 25 & 3.40 & 1.60 & 68.38 & 1.23 & 2.10 & $193 . .38$ & 303 & 32.89 & 118.15 & 14.82 & 3.84 & 14.13 & 2.64 & 12.98 \\
\hline PCS-20/08 & 153 & $<20$ & 120 & $<10$ & 438 & 14 & 2.24 & 1.41 & 55.89 & 0.80 & 1.26 & 113.33 & 200.08 & 23.48 & 89.23 & 13.50 & 4.39 & 11.47 & 2.19 & 10.61 \\
\hline BRS-07/08 & 114 & $<20$ & 78 & 23 & 310 & 7 & 2.92 & 1.7 & 81 & 1.61 & 2.35 & 600.48 & 864.51 & 88.83 & 278.42 & 28.84 & 2.19 & 36.14 & 4.85 & 17.23 \\
\hline BRS-09/08 & 246 & $<20$ & 165 & $<10$ & 567 & 8 & 4.46 & 3.41 & 138.63 & 4.8 & 4.79 & 467.18 & 823.93 & 76.18 & 254.48 & 35.61 & 2.95 & 32.91 & 5.23 & 24.85 \\
\hline BRS-10/07 & 168 & $<20$ & 107 & $<10$ & 373 & 9 & 5.17 & 3.15 & 117.33 & 2.78 & 5.28 & 380.59 & 634.33 & 62.86 & 212.1 & 30.81 & 2.37 & 26.98 & 4.4 & 20.54 \\
\hline BRS-11/08 & $<4$ & 315 & 25 & 107 & 51 & 77 & 0.27 & 1.38 & 28.24 & 1.56 & 2.68 & 3.78 & 10.51 & 1.4 & 7.45 & 2.37 & 0.86 & 1.43 & 0.55 & 4.75 \\
\hline BRS-12/07 & 139 & $<20$ & 123 & $<10$ & 425 & 5 & 1.69 & 2.6 & 110.3 & 1.04 & 4.63 & 605.75 & 949.95 & 83.97 & 290.63 & 35.81 & 2.34 & 39.11 & 5.43 & 20.47 \\
\hline BRS-13/08 & 112 & $<20$ & 102 & 37 & 343 & 5 & 1.07 & 1.99 & 101.33 & 0.93 & 2.91 & 663.07 & 880.57 & 94.86 & 298.53 & 33.5 & 2.24 & 40.12 & 5.33 & 19.62 \\
\hline BRS-14/08 & 36 & $<20$ & 19 & $<10$ & 96 & 6 & 1.11 & 1.11 & 28.5 & 0.5 & 2.83 & 25.3 & 78.59 & 5.75 & 20.75 & 4.17 & 0.95 & 3.52 & 0.97 & 6.62 \\
\hline BRS-15/08 & 34 & $<20$ & 58 & $<10$ & 214 & 10 & 2.76 & 1.95 & 81.14 & 0.79 & 4.41 & 155.5 & 276.47 & 27.9 & 99.05 & 13.29 & 2.27 & 13.39 & 2.49 & 13.13 \\
\hline & Ho & $\mathrm{Er}$ & $\mathrm{Tm}$ & $\mathrm{Yb}$ & $\mathrm{Lu}$ & $\mathrm{Hf}$ & $\mathrm{Ta}$ & W & U & \multicolumn{2}{|c|}{$\Sigma$ REE (ppm) } & & & & & & & & & \\
\hline PCS-11/08 & 2.75 & 7.83 & 1.08 & 7.13 & 0.95 & 16.75 & 1.90 & 2.91 & 2.29 & \multicolumn{2}{|c|}{715.57} & & & & & & & & & \\
\hline PCS-20/08 & 2.23 & 6.43 & 0.89 & 5.73 & 0.78 & 15.90 & 1.55 & 1.38 & 0.99 & \multicolumn{2}{|c|}{484.35} & & & & & & & & & \\
\hline BRS-07/08 & 3.45 & 12.28 & 1.31 & 8.85 & 1.16 & 16.08 & 1.82 & 155.67 & 4.13 & \multicolumn{2}{|c|}{1948.54} & & & & & & & & & \\
\hline BRS-09/08 & 5.26 & 16.85 & 2.55 & 16.99 & 2.39 & 20.42 & 3.42 & 51.12 & 11.31 & \multicolumn{2}{|c|}{1767.35} & & & & & & & & & \\
\hline BRS-10/07 & 4.25 & 13.57 & 1.96 & 13.59 & 1.95 & 18.1 & 3.31 & 53.09 & 10.41 & \multicolumn{2}{|c|}{1410.29} & & & & & & & & & \\
\hline BRS-11/08 & 1.09 & 2.82 & 0.5 & 3.3 & 0.48 & 2.08 & 0.09 & 47.77 & $<0.50$ & \multicolumn{2}{|c|}{41.30} & & & & & & & & & \\
\hline BRS-12/07 & 4.44 & 14.81 & 1.9 & 13.22 & 1.84 & 15.86 & 3.8 & 128.08 & 4.93 & \multicolumn{2}{|c|}{2069.67} & & & & & & & & & \\
\hline BRS-13/08 & 4.03 & 14.36 & 1.69 & 11.62 & 1.68 & 12.55 & 4.74 & 122.39 & 5.19 & \multicolumn{2}{|c|}{2071.20} & & & & & & & & & \\
\hline BRS-14/08 & 1.42 & 3.48 & 0.55 & 3.56 & 0.49 & 6.28 & 2.75 & 110.54 & 4.12 & \multicolumn{2}{|c|}{156.12} & & & & & & & & & \\
\hline BRS-15/08 & 2.97 & 8.83 & 1.38 & 9.43 & 1.29 & 10.27 & 2.07 & 133.78 & 3.61 & \multicolumn{2}{|c|}{627.40} & & & & & & & & & \\
\hline
\end{tabular}

Migmatite: BRS-14, BRS-15, PCS-11/8, Syenite: PCS 20/8, BRS-7/8, BRS- 9/8, BRS-10/8, BRS-12/8, BRS-13/8 Amphibolite: BRS-11/8.

Major oxides values are in \%; REE and trace element values are in ppm. 
Table 3. REE and silicate analysis chemical data (EPMA) of five samples

\begin{tabular}{|c|c|c|c|c|c|c|c|c|c|c|c|}
\hline Sample Name & & & & & & 8 (Migmati & & & & & \\
\hline Point No. & $1 / 1$ & $2 / 1$ & $4 / 1$ & $5 / 1$ & $11 / 1$ & $7 / 1$ & $12 / 1$ & $8 / 1$ & $9 / 1$ & $10 / 1$ & $14 / 1$ \\
\hline $\mathrm{Na}_{2} \mathrm{O}$ & 0.02 & 0.03 & 0.15 & 0.11 & 0.03 & 0 & 0 & 0.03 & 0.06 & 0.05 & 0.04 \\
\hline $\mathrm{MgO}$ & 0.31 & 0.34 & 0.18 & 0.31 & 0.57 & 0.16 & 0.21 & 0.02 & 0.12 & 0.04 & 0.14 \\
\hline $\mathrm{CaO}$ & 11.1 & 11.6 & 14.2 & 12 & 9.58 & 7.65 & 7.22 & 0.22 & 27.5 & 28.4 & 27.67 \\
\hline $\mathrm{MnO}$ & 0.29 & 0.33 & 0.46 & 0.15 & 0.46 & 0 & 0 & 0.02 & 0.15 & 0.11 & 0.14 \\
\hline $\mathrm{FeO}$ & 15.4 & 14.9 & 11.6 & 8.99 & 15.67 & 1.22 & 1.92 & 0.43 & 2.06 & 1.21 & 2.15 \\
\hline $\mathrm{SmO}$ & 0.21 & 0.15 & 0.34 & 0.15 & 0.19 & 0.7 & 0.67 & 0 & 0.02 & 0.03 & 0.05 \\
\hline $\mathrm{EuO}$ & 0 & 0 & 0 & 0 & 0 & 0 & 0 & 0.03 & 0.02 & 0.01 & 0 \\
\hline $\mathrm{PbO}$ & 0.09 & 0.13 & 0.05 & 0.07 & 0.07 & 0 & 0.01 & 4.81 & 0 & 0.01 & 0.03 \\
\hline $\mathrm{Al}_{2} \mathrm{O}_{3}$ & 14.3 & 14.8 & 16.9 & 17.8 & 12.9 & 6.56 & 6.7 & 0.02 & 5.73 & 3.54 & 5.81 \\
\hline $\mathrm{La}_{2} \mathrm{O}_{3}$ & 8.94 & 8.41 & 6.3 & 4.38 & 9.68 & 16.82 & 17.1 & 0.05 & 0 & 0 & 0 \\
\hline $\mathrm{Ce}_{2} \mathrm{O}_{3}$ & 11.1 & 10.7 & 8.44 & 5.25 & 12.14 & 24.51 & 22.61 & 0.04 & 0.4 & 0.09 & 0.33 \\
\hline $\mathrm{Pr}_{2} \mathrm{O}_{3}$ & 1.26 & 1.18 & 0.88 & 0.46 & 1.11 & 2.34 & 1.93 & 0 & 0.11 & 0 & 0 \\
\hline $\mathrm{Nd}_{2} \mathrm{O}_{3}$ & 2.38 & 2.32 & 2.11 & 1.14 & 2.41 & 7.03 & 6.87 & 0.09 & 0.34 & 0.02 & 0.39 \\
\hline $\mathrm{Gd}_{2} \mathrm{O}_{3}$ & 1.2 & 1.13 & 0.92 & 0.54 & 1.15 & 2.86 & 2.7 & 0.14 & 0.17 & 0 & 0.15 \\
\hline $\mathrm{Tb}_{2} \mathrm{O}_{3}$ & 0 & 0 & 0 & 0 & 0 & 0 & 0 & 0 & 0 & 0 & 0 \\
\hline $\mathrm{Dy}_{2} \mathrm{O}_{3}$ & 0.12 & 0.11 & 0.05 & 0 & 0.05 & 0.27 & 0.31 & 0.29 & 0.23 & 0.17 & 0.12 \\
\hline $\mathrm{Ho}_{2} \mathrm{O}_{3}$ & 0.03 & 0 & 0 & 0 & 0 & 0.06 & 0.09 & 0 & 0.11 & 0 & 0.11 \\
\hline $\mathrm{Er}_{2} \mathrm{O}_{3}$ & 0 & 0 & 0 & 0 & 0 & 0.03 & 0.02 & 0 & 0.12 & 0.08 & 0 \\
\hline $\mathrm{Tm}_{2} \mathrm{O}_{3}$ & 0 & 0 & 0 & 0 & 0 & 0.01 & 0.02 & 0 & 0.06 & 0.07 & 0.04 \\
\hline $\mathrm{Yb}_{2} \mathrm{O}_{3}$ & 0.13 & 0.2 & 0 & 0 & 0 & 0 & 0 & 0 & 0.2 & 0.11 & 0.07 \\
\hline $\mathrm{Lu}_{2} \mathrm{O}_{3}$ & 0.09 & 0.01 & 0 & 0.02 & 0.04 & 0.07 & 0 & 0.04 & 0.04 & 0.05 & 0.12 \\
\hline $\mathrm{SiO}_{2}$ & 30.5 & 31.7 & 33.2 & 39.6 & 29.95 & 10.31 & 10.01 & 18.48 & 30.6 & 30.3 & 30.59 \\
\hline $\mathrm{TiO}_{2}$ & 0.81 & 0.89 & 0.35 & 0.21 & 1.28 & 0.39 & 0.41 & 0.14 & 28.8 & 33.7 & 29.04 \\
\hline $\mathrm{ZrO}_{2}$ & 0 & 0 & 0 & 0 & 0 & 0 & 0 & 0 & 0 & 0 & 0 \\
\hline $\mathrm{ThO}_{2}$ & 1.5 & 1.81 & 2.18 & 0.8 & 1.49 & 3.58 & 3.15 & 70.11 & 0.08 & 0 & 0.07 \\
\hline $\mathrm{UO}_{2}$ & 0 & 0.09 & 0.03 & 0.14 & 0.01 & 0.13 & 0.23 & 0 & 0.04 & 0.01 & 0.01 \\
\hline $\mathrm{P}_{2} \mathrm{O}_{5}$ & 0 & 0 & 0 & 0 & 0 & 0 & 0 & 0 & 0 & 0 & 0 \\
\hline Total & 99.6 & 101 & 98.4 & 92.1 & 98.78 & 84.7 & 82.18 & 94.96 & 96.9 & 97.9 & 97.07 \\
\hline Comment & allanite & allanite & allanite & allanite & allanite & britholite & britholite & thorite & titanite & titanite & titanite \\
\hline Sample Name & & & & & PCS & 8 (Amet G & nite) & & & & \\
\hline Point No. & $1 / 1$ & $2 / 1$ & $4 / 1$ & $5 / 1$ & $6 / 1$ & $7 / 1$ & $8 / 1$ & $9 / 1$ & $10 / 1$ & $12 / 1$ & $11 / 1$ \\
\hline $\mathrm{Na}_{2} \mathrm{O}$ & 0.03 & 0.01 & 0.02 & 0 & 0.04 & 0.04 & 0.02 & 0.01 & 0.02 & 0.02 & 0.01 \\
\hline $\mathrm{MgO}$ & 0.37 & 0.15 & 0.27 & 0.18 & 0.35 & 0.28 & 0.13 & 0.24 & 0.09 & 0 & 0.01 \\
\hline $\mathrm{CaO}$ & 14.3 & 19.4 & 18.1 & 19.8 & 15.3 & 15.4 & 19.7 & 18.9 & 20.6 & 27.8 & 23.3 \\
\hline $\mathrm{MnO}$ & 0.31 & 0.19 & 0.24 & 0.28 & 0.28 & 0.19 & 0.19 & 0.15 & 0.26 & 0.13 & 0.17 \\
\hline $\mathrm{FeO}$ & 13.2 & 12.1 & 12.3 & 12.3 & 12.5 & 12.9 & 12 & 12.1 & 11.8 & 1.09 & 12.2 \\
\hline $\mathrm{SmO}$ & 0.25 & 0.14 & 0.19 & 0.09 & 0.46 & 0.26 & 0.17 & 0.09 & 0.05 & 0.07 & 0 \\
\hline $\mathrm{EuO}$ & 0 & 0 & 0 & 0.09 & 0 & 0 & 0 & 0 & 0.06 & 0.04 & 0.1 \\
\hline $\mathrm{PbO}$ & 0 & 0 & 0.02 & 0.01 & 0.02 & 0 & 0 & 0.04 & 0.07 & 0 & 0 \\
\hline $\mathrm{Al}_{2} \mathrm{O}_{3}$ & 18.5 & 21.5 & 20.2 & 21.2 & 20.8 & 19.3 & 22 & 21.8 & 21.9 & 2.13 & 23.4 \\
\hline $\mathrm{La}_{2} \mathrm{O}_{3}$ & 6.12 & 2.37 & 2.22 & 2.18 & 4.01 & 4.06 & 2.12 & 3.78 & 1.54 & 0 & 0 \\
\hline $\mathrm{Ce}_{2} \mathrm{O}_{3}$ & 8.75 & 3.24 & 4.27 & 3.09 & 7.4 & 7.48 & 3.05 & 4.38 & 2.09 & 0.13 & 0.04 \\
\hline $\mathrm{Pr}_{2} \mathrm{O}_{3}$ & 0.93 & 0.28 & 0.59 & 0.38 & 1.1 & 0.97 & 0.16 & 0.34 & 0.06 & 0 & 0 \\
\hline $\mathrm{Nd}_{2} \mathrm{O}_{3}$ & 2.87 & 1.54 & 2.17 & 1.27 & 3.27 & 3.27 & 1.41 & 1.09 & 0.78 & 0.12 & 0 \\
\hline $\mathrm{Gd}_{2} \mathrm{O}_{3}$ & 0.78 & 0.32 & 0.54 & 0.36 & 0.79 & 1.03 & 0.4 & 0.33 & 0.38 & 0.02 & 0 \\
\hline $\mathrm{Tb}_{2} \mathrm{O}_{3}$ & 0 & 0 & 0 & 0 & 0 & 0 & 0 & 0 & 0 & 0 & 0 \\
\hline $\mathrm{Dy}_{2} \mathrm{O}_{3}$ & 0.08 & 0.09 & 0.15 & 0 & 0.13 & 0.1 & 0.08 & 0 & 0.2 & 0 & 0.18 \\
\hline $\mathrm{Ho}_{2} \mathrm{O}_{3}$ & 0.03 & 0.01 & 0.03 & 0.02 & 0.06 & 0.08 & 0 & 0 & 0 & 0.07 & 0 \\
\hline
\end{tabular}


Table 3. Continued

\begin{tabular}{|c|c|c|c|c|c|c|c|c|c|c|c|}
\hline \multirow{2}{*}{$\begin{array}{c}\text { Sample Name } \\
\text { Point No. }\end{array}$} & \multicolumn{11}{|c|}{ PCS 1-8 (Amet Granite) } \\
\hline & $1 / 1$ & $2 / 1$ & $4 / 1$ & $5 / 1$ & $6 / 1$ & $7 / 1$ & $8 / 1$ & $9 / 1$ & $10 / 1$ & $12 / 1$ & $11 / 1$ \\
\hline $\mathrm{Er}_{2} \mathrm{O}_{3}$ & 0 & 0 & 0.02 & 0 & 0 & 0 & 0 & 0 & 0 & 0.11 & 0 \\
\hline $\mathrm{Tm}_{2} \mathrm{O}_{3}$ & 0 & 0.06 & 0 & 0 & 0 & 0 & 0 & 0 & 0 & 0 & 0 \\
\hline $\mathrm{Yb}_{2} \mathrm{O}_{3}$ & 0.13 & 0.07 & 0.05 & 0 & 0 & 0 & 0 & 0.14 & 0.07 & 0 & 0.03 \\
\hline $\mathrm{Lu}_{2} \mathrm{O}_{3}$ & 0 & 0 & 0.1 & 0.04 & 0 & 0 & 0.02 & 0.01 & 0 & 0.03 & 0 \\
\hline $\mathrm{SiO}_{2}$ & 33.2 & 35.1 & 34 & 35 & 36.1 & 33 & 36 & 35.2 & 35.6 & 29.6 & 36.9 \\
\hline $\mathrm{TiO}_{2}$ & 0.18 & 0.17 & 0.19 & 0.2 & 0.17 & 0.16 & 0.14 & 0.16 & 0.12 & 35 & 0.1 \\
\hline $\mathrm{ZrO}_{2}$ & 0 & 0 & 0 & 0 & 0 & 0 & 0 & 0 & 0 & 0 & 0 \\
\hline $\mathrm{ThO}_{2}$ & 0.14 & 0.12 & 0.04 & 0.06 & 0.04 & 0.03 & 0.05 & 0.09 & 0.05 & 0 & 0 \\
\hline $\mathrm{UO}_{2}$ & 0.05 & 0 & 0 & 0 & 0.02 & 0.06 & 0.04 & 0.09 & 0 & 0 & 0.01 \\
\hline $\mathrm{P}_{2} \mathrm{O}_{5}$ & 0 & 0 & 0 & 0.01 & 0 & 0.02 & 0 & 0.01 & 0.01 & 0.02 & 0 \\
\hline Total & 100 & 96.9 & 95.6 & 96.5 & 103 & 98.6 & 97.7 & 98.9 & 95.8 & 96.4 & 96.4 \\
\hline Comment & allanite & allanite & allanite & allanite & allanite & allanite & allanite & allanite & allanite & allanite & epidote \\
\hline Sample Name & \multicolumn{11}{|c|}{ 55B-8 (syenite/alkali feldspar syenite) } \\
\hline Point No. & $2 / 1$ & \multicolumn{2}{|r|}{$5 / 1$} & $7 / 1$ & \multicolumn{2}{|l|}{$1 / 1$} & $3 / 1$ & $4 / 1$ & \multicolumn{2}{|l|}{$8 / 1$} & $6 / 1$ \\
\hline $\mathrm{Na}_{2} \mathrm{O}$ & 0.45 & \multicolumn{2}{|r|}{0.03} & 0.00 & \multicolumn{2}{|l|}{0.07} & 0.13 & 0.06 & \multicolumn{2}{|l|}{0.03} & 0.00 \\
\hline $\mathrm{MgO}$ & 0.28 & \multicolumn{2}{|r|}{0.01} & 0.03 & \multicolumn{2}{|l|}{0.10} & 0.16 & 0.11 & \multicolumn{2}{|l|}{0.09} & 0.01 \\
\hline $\mathrm{CaO}$ & 11.99 & \multicolumn{2}{|r|}{55.54} & 55.86 & \multicolumn{2}{|l|}{7.13} & 8.42 & 26.79 & \multicolumn{2}{|l|}{27.14} & 0.00 \\
\hline $\mathrm{MnO}$ & 0.27 & \multicolumn{2}{|r|}{0.11} & 0.01 & \multicolumn{2}{|l|}{0.00} & 0.00 & 0.21 & \multicolumn{2}{|l|}{0.20} & 0.00 \\
\hline $\mathrm{FeO}$ & 10.03 & \multicolumn{2}{|r|}{0.00} & 0.04 & \multicolumn{2}{|l|}{4.77} & 6.02 & 1.82 & \multicolumn{2}{|l|}{1.79} & 0.11 \\
\hline $\mathrm{SmO}$ & 0.26 & & 0.00 & 0.02 & 0.67 & & 0.83 & 0.00 & 0.00 & & 0.04 \\
\hline $\mathrm{EuO}$ & 0.00 & & 0.00 & 0.00 & 0.00 & & 0.00 & 0.06 & 0.05 & & 0.00 \\
\hline $\mathrm{PbO}$ & 0.06 & & 0.00 & 0.00 & 0.04 & & 0.06 & 0.01 & 0.06 & & 0.05 \\
\hline $\mathrm{Al}_{2} \mathrm{O}_{3}$ & 17.17 & & 0.00 & 0.00 & 8.71 & & 8.92 & 8.46 & 8.25 & & 0.01 \\
\hline $\mathrm{La}_{2} \mathrm{O}_{3}$ & 4.45 & & 0.03 & 0.01 & 13.24 & & 10.10 & 0.00 & 0.00 & & 0.00 \\
\hline $\mathrm{Ce}_{2} \mathrm{O}_{3}$ & 6.97 & & 0.01 & 0.01 & 18.22 & & 16.00 & 0.14 & 0.09 & & 0.03 \\
\hline $\mathrm{Pr}_{2} \mathrm{O}_{3}$ & 0.78 & & 0.00 & 0.00 & 2.11 & & 2.02 & 0.00 & 0.00 & & 0.00 \\
\hline $\mathrm{Nd}_{2} \mathrm{O}_{3}$ & 1.76 & & 0.00 & 0.09 & 5.50 & & 5.29 & 0.00 & 0.01 & & 0.03 \\
\hline $\mathrm{Gd}_{2} \mathrm{O}_{3}$ & 1.05 & & 0.00 & 0.05 & 2.13 & & 2.12 & 0.15 & 0.00 & & 0.00 \\
\hline $\mathrm{Tb}_{2} \mathrm{O}_{3}$ & 0.00 & & 0.00 & 0.00 & 0.00 & & 0.00 & 0.00 & 0.00 & & 0.00 \\
\hline $\mathrm{Dy}_{2} \mathrm{O}_{3}$ & 0.47 & & 0.26 & 0.00 & 0.35 & & 0.46 & 0.33 & 0.22 & & 0.14 \\
\hline $\mathrm{Ho}_{2} \mathrm{O}_{3}$ & 0.08 & & 0.00 & 0.00 & 0.12 & & 0.11 & 0.00 & 0.09 & & 0.00 \\
\hline $\mathrm{Er} 2 \mathrm{O}_{3}$ & 0.00 & & 0.14 & 0.00 & 0.00 & & 0.09 & 0.07 & 0.17 & & 0.00 \\
\hline $\mathrm{Tm}_{2} \mathrm{O} 3$ & 0.03 & & 0.04 & 0.00 & 0.19 & & 0.19 & 0.00 & 0.00 & & 0.13 \\
\hline $\mathrm{Yb}_{2} \mathrm{O}_{3}$ & 0.06 & & 0.00 & 0.00 & 0.00 & & 0.12 & 0.23 & 0.19 & & 0.13 \\
\hline $\mathrm{Lu}_{2} \mathrm{O}_{3}$ & 0.00 & & 0.00 & 0.00 & 0.00 & & 0.00 & 0.09 & 0.05 & & 0.00 \\
\hline $\mathrm{SiO}_{2}$ & 34.79 & & 0.08 & 0.10 & 17.60 & & 20.02 & 29.83 & 30.38 & & 31.18 \\
\hline $\mathrm{TiO}_{2}$ & 0.33 & & 0.02 & 0.02 & 0.18 & & 0.25 & 24.64 & 25.43 & & 0.03 \\
\hline $\mathrm{ZrO}_{2}$ & 0.00 & & 0.00 & 0.00 & 0.00 & & 0.00 & 0.00 & 0.00 & & 59.99 \\
\hline $\mathrm{ThO}_{2}$ & 1.77 & & 0.00 & 0.03 & 3.56 & & 5.77 & 0.00 & 0.05 & & 0.08 \\
\hline $\mathrm{UO}_{2}$ & 0.08 & & 0.01 & 0.01 & 0.06 & & 0.13 & 0.04 & 0.00 & & 0.09 \\
\hline $\mathrm{P}_{2} \mathrm{O}_{5}$ & 0.00 & & 38.40 & 37.72 & 0.00 & & 0.00 & 0.00 & 0.00 & & 0.35 \\
\hline Total & 93.13 & & 94.68 & 94.00 & 84.75 & & 87.21 & 93.04 & 94.29 & & 92.40 \\
\hline Comment & allanite & & apatite & apatite & britholite & & ritholite & titanite & titanite & & zircon \\
\hline
\end{tabular}

chosen and thin polished sections were prepared for EPMA studies. Five thin polished sections of allanite bearing migmatite gneiss (1 no.), Amet granite (2 no.) and syenite-alkali feldspar syenite ( 2 no.) were studied for their silicate and REE content and analysis of mineral phases (Table 3). Cation proportions of the minerals studied (five samples) are given in Table 4 and mineral formula calculated on the basis of $13 \mathrm{O}$ for allanite for the same five samples is given in Table 5 . Total 18 rock samples were collected for silicate and REE analysis 
Table 3. Continued

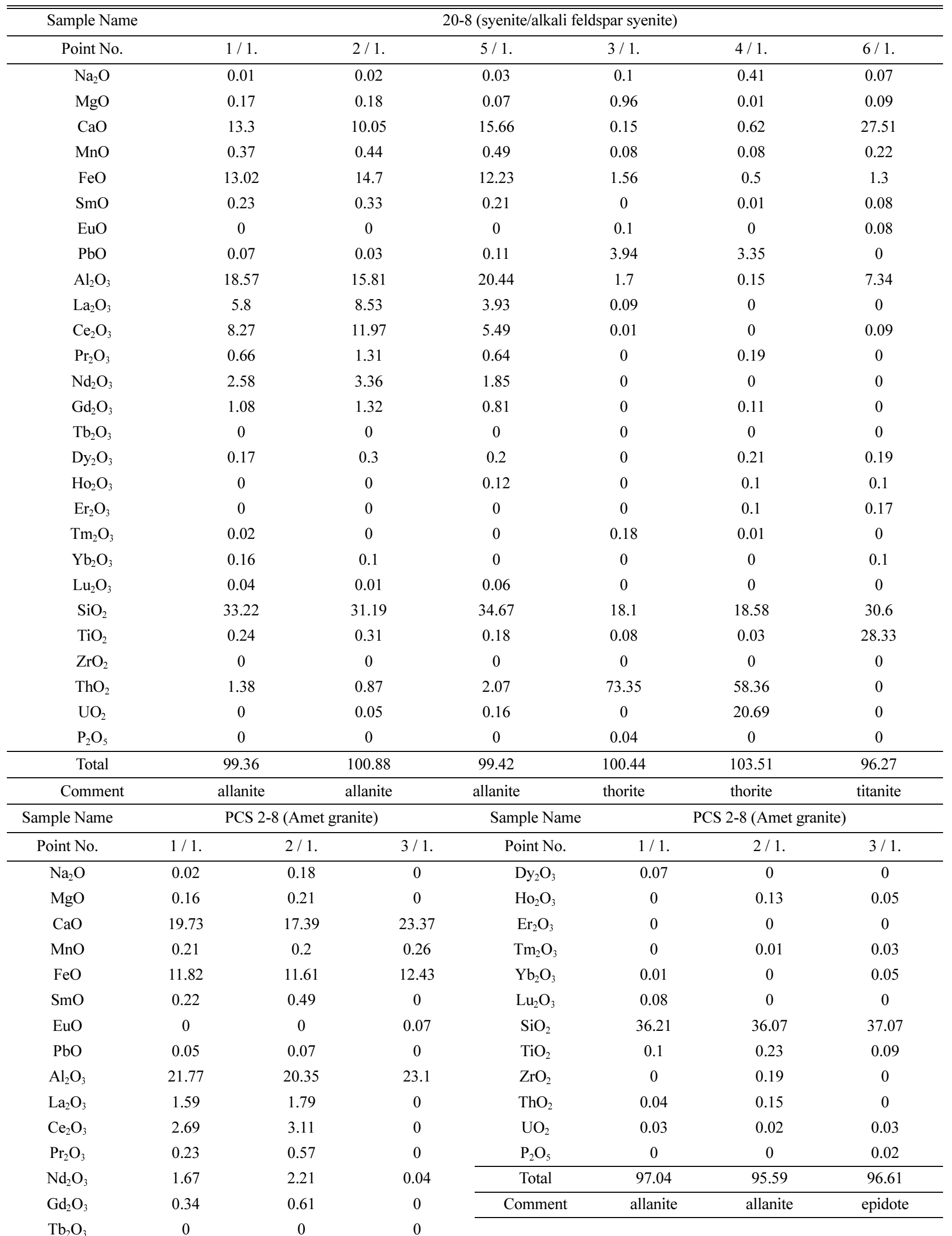


Table 4. Cation proportions of allanite calculated from EPMA chemical data

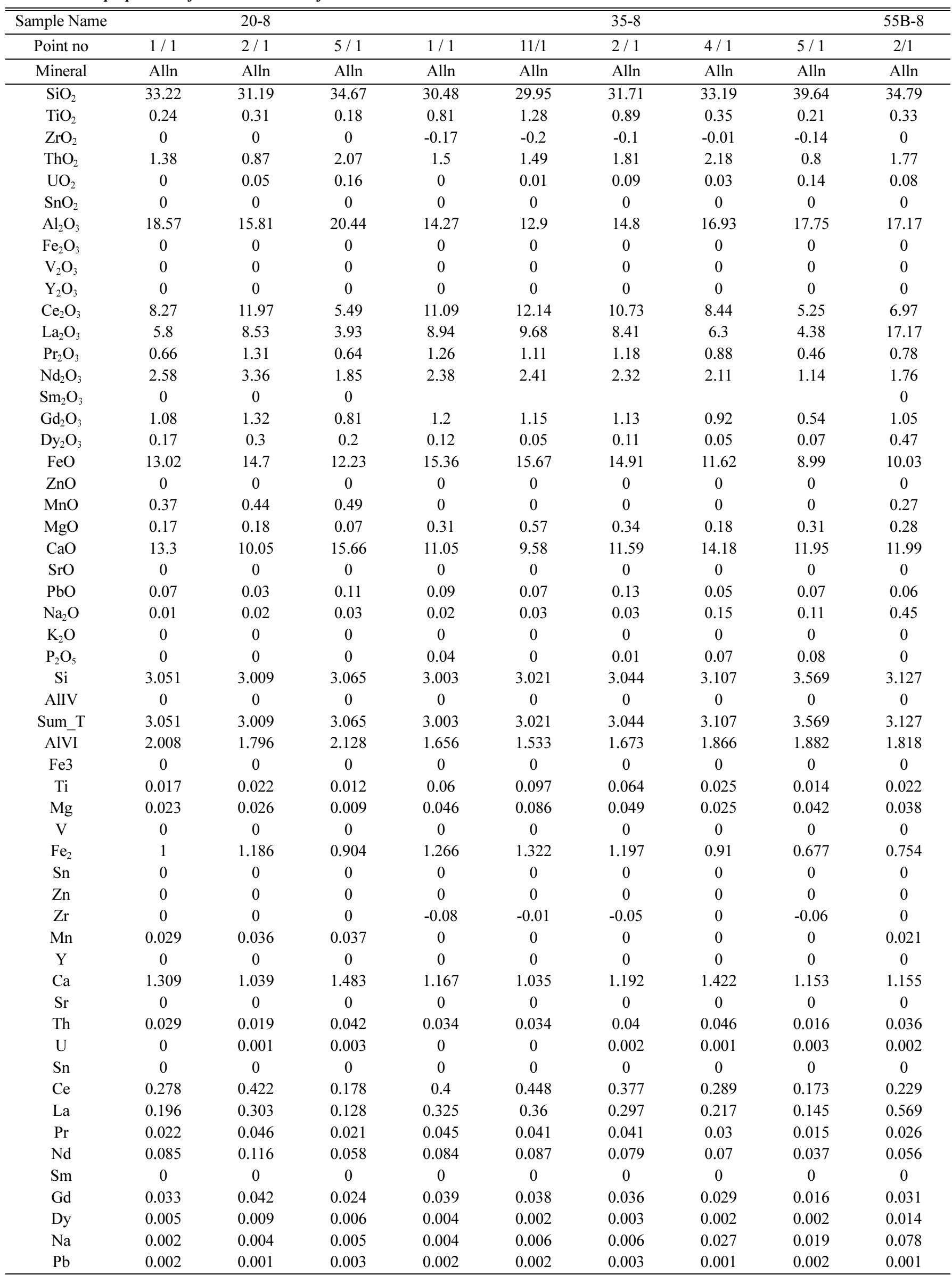


Table 4. Continued

\begin{tabular}{|c|c|c|c|c|c|c|c|c|c|}
\hline Sample Name & & & & & CS $1-8$ & & & & \\
\hline Point No. & $1 / 1$ & $10 / 1$ & $11 / 1$ & $12 / 1$ & $2 / 1$ & $4 / 1$ & $5 / 1$ & $6 / 1$ & $7 / 1$ \\
\hline Mineral & Alln & Alln & Alln & Alln & Alln & Alln & Alln & Alln & Alln \\
\hline $\mathrm{SiO}_{2}$ & 33.17 & 35.6 & 36.88 & 29.55 & 35.1 & 33.97 & 34.95 & 36.11 & 32.99 \\
\hline $\mathrm{TiO}_{2}$ & 0.18 & 0.12 & 0.1 & 35.01 & 0.17 & 0.19 & 0.2 & 0.17 & 0.16 \\
\hline $\mathrm{ZrO}_{2}$ & 0 & 0 & 0 & 0 & 0 & 0 & 0 & 0 & 0 \\
\hline $\mathrm{ThO}_{2}$ & 0.14 & 0.05 & 0 & 0 & 0.12 & 0.04 & 0.06 & 0.04 & 0.03 \\
\hline $\mathrm{UO}_{2}$ & 0.05 & 0 & 0.01 & 0 & 0 & 0 & 0 & 0.02 & 0.06 \\
\hline $\mathrm{SnO} 2$ & 0 & 0 & 0 & 0 & 0 & 0 & 0 & 0 & 0 \\
\hline $\mathrm{Al}_{2} \mathrm{O}_{3}$ & 18.45 & 21.93 & 23.42 & 2.13 & 21.52 & 20.15 & 21.2 & 20.83 & 19.31 \\
\hline $\mathrm{Fe}_{2} \mathrm{O}_{3}$ & 0 & 0 & 0 & 0 & 0 & 0 & 0 & 0 & 0 \\
\hline $\mathrm{V}_{2} \mathrm{O}_{3}$ & 0 & 0 & 0 & 0 & 0 & 0 & 0 & 0 & 0 \\
\hline $\mathrm{Y}_{2} \mathrm{O}_{3}$ & 0 & 0 & 0 & 0 & 0 & 0 & 0 & 0 & 0 \\
\hline $\mathrm{Ce}_{2} \mathrm{O}_{3}$ & 8.75 & 2.09 & 0.04 & 0.13 & 3.24 & 4.27 & 3.09 & 7.4 & 7.48 \\
\hline $\mathrm{La}_{2} \mathrm{O}_{3}$ & 6.12 & 1.54 & 0 & 0 & 2.37 & 2.22 & 2.18 & 4.01 & 4.06 \\
\hline $\mathrm{Nd}_{2} \mathrm{O}_{3}$ & 2.87 & 0.78 & 0 & 0.12 & 1.54 & 2.17 & 1.27 & 3.27 & 3.27 \\
\hline $\mathrm{Sm}_{2} \mathrm{O}_{3}$ & & & & & & & & & \\
\hline $\mathrm{Gd}_{2} \mathrm{O}_{3}$ & 0.78 & 0.38 & 0 & 0.02 & 0.32 & 0.54 & 0.36 & 0.79 & 1.03 \\
\hline $\mathrm{Dy}_{2} \mathrm{O}_{3}$ & 0.08 & 0.2 & 0.18 & 0 & 0.09 & 0.15 & 0 & 0.13 & 0.1 \\
\hline $\mathrm{FeO}$ & 13.18 & 11.84 & 12.23 & 1.09 & 12.12 & 12.25 & 12.27 & 12.47 & 12.9 \\
\hline $\mathrm{ZnO}$ & & & & & & & & & \\
\hline $\mathrm{MnO}$ & 0.31 & 0.26 & 0.17 & 0.13 & 0.19 & 0.24 & 0.28 & 0.28 & 0.19 \\
\hline $\mathrm{MgO}$ & 0.37 & 0.09 & 0.01 & 0 & 0.15 & 0.27 & 0.18 & 0.35 & 0.28 \\
\hline $\mathrm{CaO}$ & 14.3 & 20.58 & 23.26 & 27.83 & 19.44 & 18.11 & 19.82 & 15.31 & 15.39 \\
\hline $\mathrm{SrO}$ & 0 & 0 & 0 & 0 & 0 & 0 & 0 & 0 & 0 \\
\hline $\mathrm{PbO}$ & 0 & 0.07 & 0 & 0 & 0 & 0.02 & 0.01 & 0.02 & 0 \\
\hline $\mathrm{Na}_{2} \mathrm{O}$ & 0.03 & 0.02 & 0.01 & 0.02 & 0.01 & 0.02 & 0 & 0.04 & 0.04 \\
\hline $\mathrm{K}_{2} \mathrm{O}$ & 0 & 0 & 0 & 0 & 0 & 0 & 0 & 0 & 0 \\
\hline AlIV & 0 & 0 & 0 & 0.214 & 0 & 0 & 0 & 0 & 0.002 \\
\hline Sum_T & 3.023 & 3.063 & 3.058 & 2.734 & 3.043 & 3.043 & 3.04 & 3.09 & 3 \\
\hline AlVI & 1.98 & 2.222 & 2.287 & 0 & 2.197 & 2.126 & 2.172 & 2.099 & 2.065 \\
\hline $\mathrm{Fe}_{3}$ & 0 & 0 & 0 & 0 & 0 & 0 & 0 & 0 & 0 \\
\hline $\mathrm{Ti}$ & 0.012 & 0.008 & 0.006 & 2.246 & 0.011 & 0.013 & 0.013 & 0.011 & 0.011 \\
\hline $\mathrm{Mg}$ & 0.05 & 0.012 & 0.001 & 0 & 0.019 & 0.036 & 0.023 & 0.045 & 0.038 \\
\hline $\mathrm{V}$ & 0 & 0 & 0 & 0 & 0 & 0 & 0 & 0 & 0 \\
\hline $\mathrm{Fe}_{2}$ & 1.004 & 0.852 & 0.848 & 0.078 & 0.879 & 0.918 & 0.893 & 0.892 & 0.98 \\
\hline $\mathrm{Sn}$ & 0 & 0 & 0 & 0 & 0 & 0 & 0 & 0 & 0 \\
\hline $\mathrm{Zn}$ & 0 & 0 & 0 & 0 & 0 & 0 & 0 & 0 & 0 \\
\hline $\mathrm{Zr}$ & 0 & 0 & 0 & 0 & 0 & 0 & 0 & 0 & 0 \\
\hline $\mathrm{Mn}$ & 0.024 & 0.019 & 0.012 & 0.009 & 0.014 & 0.018 & 0.021 & 0.02 & 0.015 \\
\hline $\mathrm{Y}$ & 0 & 0 & 0 & 0 & 0 & 0 & 0 & 0 & 0 \\
\hline $\mathrm{Ca}$ & 1.396 & 1.897 & 2.066 & 2.543 & 1.806 & 1.738 & 1.847 & 1.404 & 1.499 \\
\hline $\mathrm{Sr}$ & 0 & 0 & 0 & 0 & 0 & 0 & 0 & 0 & 0 \\
\hline Th & 0.003 & 0.001 & 0 & 0 & 0.002 & 0.001 & 0.001 & 0.001 & 0.001 \\
\hline $\mathrm{U}$ & 0.001 & 0 & 0 & 0 & 0 & 0 & 0 & 0 & 0.001 \\
\hline $\mathrm{Sn}$ & 0 & 0 & 0 & 0 & 0 & 0 & 0 & 0 & 0 \\
\hline $\mathrm{Ce}$ & 0.292 & 0.066 & 0.001 & 0.004 & 0.103 & 0.14 & 0.098 & 0.232 & 0.249 \\
\hline $\mathrm{La}$ & 0.205 & 0.049 & 0 & 0 & 0.076 & 0.073 & 0.07 & 0.126 & 0.136 \\
\hline $\operatorname{Pr}$ & 0.031 & 0.002 & 0 & 0 & 0.009 & 0.019 & 0.012 & 0.034 & 0.032 \\
\hline $\mathrm{Nd}$ & 0.093 & 0.024 & 0 & 0.004 & 0.048 & 0.069 & 0.039 & 0.1 & 0.106 \\
\hline $\mathrm{Sm}$ & 0 & 0 & 0 & 0 & 0 & 0 & 0 & 0 & 0 \\
\hline $\mathrm{Gd}$ & 0.024 & 0.011 & 0 & 0.001 & 0.009 & 0.016 & 0.01 & 0.022 & 0.031 \\
\hline Dy & 0.002 & 0.006 & 0.005 & 0 & 0.003 & 0.004 & 0 & 0.004 & 0.003 \\
\hline $\mathrm{Na}$ & 0.005 & 0.003 & 0.002 & 0.003 & 0.002 & 0.003 & 0 & 0.007 & 0.007 \\
\hline $\mathrm{Pb}$ & 0 & 0.002 & 0 & 0 & 0 & 0 & 0 & 0 & 0 \\
\hline $\mathrm{K}$ & 0 & 0 & 0 & 0 & 0 & 0 & 0 & 0 & 0 \\
\hline
\end{tabular}


Table 4. Continued

\begin{tabular}{|c|c|c|c|c|}
\hline "Sample Name & \multicolumn{2}{|c|}{ PCS 1-8 } & \multicolumn{2}{|c|}{ PCS 2-8 } \\
\hline Point No. & $8 / 1$ & $9 / 1$ & $1 / 1$ & $2 / 1$ \\
\hline Mineral & Alln & Alln & Alln & Alln \\
\hline $\mathrm{SiO}_{2}$ & 36.02 & 35.2 & 36.21 & 36.07 \\
\hline $\mathrm{TiO}_{2}$ & 0.14 & 0.16 & 0.1 & 0.23 \\
\hline $\mathrm{ZrO}_{2}$ & 0 & 0 & 0 & 0.19 \\
\hline $\mathrm{ThO}_{2}$ & 0.05 & 0.09 & 0.04 & 0.15 \\
\hline $\mathrm{UO}_{2}$ & 0.04 & 0.09 & 0.03 & 0.02 \\
\hline $\mathrm{SnO}_{2}$ & 0 & 0 & 0 & 0 \\
\hline $\mathrm{Al}_{2} \mathrm{O}_{3}$ & 22 & 21.78 & 21.77 & 20.35 \\
\hline $\mathrm{Fe}_{2} \mathrm{O}_{3}$ & 0 & 0 & 0 & 0 \\
\hline $\mathrm{V}_{2} \mathrm{O}_{3}$ & 0 & 0 & 0 & 0 \\
\hline $\mathrm{Y}_{2} \mathrm{O}_{3}$ & 0 & 0 & 0 & 0 \\
\hline $\mathrm{Ce}_{2} \mathrm{O}_{3}$ & 3.05 & 4.38 & 2.69 & 3.11 \\
\hline $\mathrm{La}_{2} \mathrm{O}_{3}$ & 2.12 & 3.78 & 1.59 & 1.79 \\
\hline $\operatorname{Pr} 2 \mathrm{O}_{3}$ & 0.16 & 0.34 & 0.23 & 0.57 \\
\hline $\mathrm{Nd}_{2} \mathrm{O}_{3}$ & 1.41 & 1.09 & 1.67 & 2.21 \\
\hline $\mathrm{Sm}_{2} \mathrm{O}_{3}$ & 0 & 0 & & \\
\hline $\mathrm{Gd}_{2} \mathrm{O}_{3}$ & 0.4 & 0.33 & 0.34 & 0.61 \\
\hline $\mathrm{Dy}_{2} \mathrm{O}_{3}$ & 0.08 & 0 & 0.07 & 0 \\
\hline $\mathrm{FeO}$ & 11.99 & 12.05 & 11.82 & 11.61 \\
\hline $\mathrm{ZnO}$ & 0 & 0 & 0 & 0 \\
\hline $\mathrm{MnO}$ & 0.19 & 0.15 & 0.21 & 0.2 \\
\hline $\mathrm{MgO}$ & 0.13 & 0.24 & 0.16 & 0.21 \\
\hline $\mathrm{CaO}$ & 19.69 & 18.9 & 19.73 & 17.39 \\
\hline $\mathrm{SrO}$ & 0 & 0 & 0 & 0 \\
\hline $\mathrm{PbO}$ & 0 & 0.04 & 0.05 & 0.07 \\
\hline $\mathrm{Na}_{2} \mathrm{O}$ & 0.02 & 0.01 & 0.02 & 0.18 \\
\hline $\mathrm{K}_{2} \mathrm{O}$ & 0 & 0 & 0 & 0 \\
\hline $\mathrm{P}_{2} \mathrm{O}_{5}$ & 0 & 0.01 & 0 & 0 \\
\hline $\mathrm{Si}$ & 3.071 & 3.024 & 3.097 & 3.17 \\
\hline AlIV & 0 & 0 & 0 & 0 \\
\hline Sum_T & 3.071 & 3.024 & 3.097 & 3.17 \\
\hline AlVI & 2.209 & 2.203 & 2.193 & 2.106 \\
\hline $\mathrm{Fe}_{3}$ & 0 & 0 & 0 & 0 \\
\hline $\mathrm{Ti}$ & 0.009 & 0.01 & 0.006 & 0.015 \\
\hline $\mathrm{Mg}$ & 0.017 & 0.031 & 0.02 & 0.028 \\
\hline V & 0 & 0 & 0 & 0 \\
\hline $\mathrm{Fe}_{2}$ & 0.855 & 0.866 & 0.845 & 0.853 \\
\hline $\mathrm{Sn}$ & 0 & 0 & 0 & 0 \\
\hline $\mathrm{Zn}$ & 0 & 0 & 0 & 0 \\
\hline $\mathrm{Zr}$ & 0 & 0 & 0 & 0.008 \\
\hline $\mathrm{Mn}$ & 0.014 & 0.011 & 0.015 & 0.015 \\
\hline $\mathrm{Y}$ & 0 & 0 & 0 & 0 \\
\hline $\mathrm{Ca}$ & 1.799 & 1.739 & 1.808 & 1.637 \\
\hline $\mathrm{Sr}$ & 0 & 0 & 0 & 0 \\
\hline Th & 0.001 & 0.002 & 0.001 & 0.003 \\
\hline $\mathrm{U}$ & 0.001 & 0.002 & 0.001 & 0 \\
\hline $\mathrm{Sn}$ & 0 & 0 & 0 & 0 \\
\hline $\mathrm{Ce}$ & 0.095 & 0.138 & 0.084 & 0.1 \\
\hline $\mathrm{La}$ & 0.067 & 0.12 & 0.05 & 0.058 \\
\hline $\operatorname{Pr}$ & 0.005 & 0.011 & 0.007 & 0.018 \\
\hline $\mathrm{Nd}$ & 0.043 & 0.033 & 0.051 & 0.069 \\
\hline $\mathrm{Sm}$ & 0 & 0 & 0 & 0 \\
\hline $\mathrm{Gd}$ & 0.011 & 0.009 & 0.01 & 0.018 \\
\hline Dy & 0.002 & 0 & 0.002 & 0 \\
\hline $\mathrm{Na}$ & 0.003 & 0.002 & 0.003 & 0.031 \\
\hline $\mathrm{Pb}$ & 0 & 0.001 & 0.001 & 0.002 \\
\hline
\end{tabular}

through XRF and ICPMS methods. Out of these, eight samples were of Amet Granite (Table 1), three samples of migmatite gneiss, six rock samples of syenite-alkali feldspar syenite and one sample of amphibolite (Table 2).

\section{Analytical Methods}

The samples thus collected were crushed, sieved, coned and quartered, then analysed for major, trace and REE at Chemical Lab of G.S.I, Western Region, Jaipur, India. Major and trace elements were analysed, using PANalytical Axios model X-ray fluorescence (XRF) spectrometers. The rock powder was ground to -200 mesh size, pressed with binder Perspex in 5\% Acetone at 20 ton $\mathrm{P}$ and pressed to form pellets. The instrument is calibrated with standards of granite and other rocks and the sample pellets were then run against standards calibrated.

The rocks samples were analysed for their silicate and REE chemistry at the GSI WR, Jaipur, NABL accredited (ISO/IEC17025: 2005) chemical laboratory. The standards used are made of solution from SRMs which are run to calibrate the instrument. The different SRM used are as follows: BE-N (basalt), DR-N (diorite), SY-3 (syenite), GS-N (granite).

Five thin polished sections of allanite bearing migmatite gneiss and monzonite-syenite/alkali feldspar syenite were studied by EPMA at National Centre of Excellence in Geoscience Research (NCEGR), GSI, Bengaluru, India for detailed mineral chemistry. These 5 no. samples were carbon coated (up to $60 \mathrm{n} \mu \mathrm{m}$ ) and analyzed for silicate chemistry and for REE.

\section{Silicate and Oxide Settings for Epma}

Silicate and oxide mineral phase analyses were performed using a CAMECA SX-100 electron microprobe analyser. The EMP is equipped with five wavelength dispersive spectrometers (WDS), e.g., WDS 1 (TAP crystal), 2 (PET crystal) and 4 (TAP crystal) built-in with low-pressure detectors, and WDS 3 (LPET crystal) and WDS 5 (LIF) built-in with high pressure detectors (all detectors use P-10 gas). Poly-propylene separation windows are used with WDS 1, 2 and 4 and Mylar windows are used with WDS 3 and 5. Calibration, overlap correction and quantifications are performed with the CAMECA SX100 Peak Sight-Geo Quanta software package. Pulse-height analysers (PHAs) adjusted by selecting the differential auto mode for all the elements. Matrix effects are eliminated by using the X-PHI correction method proposed by Merlet $(1992,1994)$. Silicate and oxide phase are analysed using $15 \mathrm{kV}$ acceleration voltage and $15 \mathrm{nA}$ beam current with $1 \mu \mathrm{m}$ size beam. The signals used are $\mathrm{Na} \mathrm{Ka}, \mathrm{Si} \mathrm{Ka}, \mathrm{Mg}$ $\mathrm{Ka}, \mathrm{Al} \mathrm{Ka}, \mathrm{K} \mathrm{Ka}$, Ca Ka, Ti Ka, Cr Ka, Fe Ka, Mn Ka for calibration of elements using natural standards for $\mathrm{Na}, \mathrm{Si}, \mathrm{Ca}, \mathrm{K}, \mathrm{Fe}, \mathrm{Mg}, \mathrm{Mn}$, synthetic standards for $\mathrm{Al}, \mathrm{Ti}, \mathrm{Cr}$. Standards used are jadeite for $\mathrm{Na}$, wollastonite for $\mathrm{Si}, \mathrm{Ca}$, diopside for $\mathrm{Mg}$, corundum for $\mathrm{Al}$, orthoclase for $\mathrm{K}$, rutile for $\mathrm{Ti}$, chromite for $\mathrm{Cr}$, haematite for $\mathrm{Fe}$ and rhodonite for $\mathrm{Mn}$. The counting time for peak measurement $10 \mathrm{~s}$ and with half of the peak measurement time allotted for background measurement. Precision of probe analysis is $\pm 1 \%$ for total analysis and $0.5 \%$ S.D. individual element wise. 
Table 5. Mineral formula of allanite based on $13(\mathrm{O}, \mathrm{OH})$ for five EPMA analysed samples

\begin{tabular}{|c|c|}
\hline Sample Name & Mineral Formula \\
\hline \multirow{5}{*}{$35-8$} & 1/1. Ca1.164Ce*0.894 Th0.034 Mn0.024 Al1.648 Fe2+1.262 Ti0.06 Mg0.045 Si2.996 O12 (OH) -0.004 Na replaced Ca \\
\hline & 11/1. Ca1.031Ce*0.971 Th0.034 Mn0.039 Al1.525 Fe2+1.316 Ti0.97 Mg0.085 Si3.007 O12 (OH) $-0.006 \mathrm{Na}$ replaced Ca \\
\hline & 2/1. Ca1.189Ce*0.832 Th0.039 Mn0.027 Al1.668 Fe2+1.194 Ti0.064 Mg0.049 Si3.036 O12 (OH) -0.006 Na replaced Ca \\
\hline & 4/1. Ca1.419Ce*0.635 Th0.046 Mn0.036 Al1.863 Fe2+0.908 Ti0.025 Mg0.025 Si3.101 O12 (OH) -0.027 Na replaced Ca \\
\hline & 5/1. Ca1.152Ce*0.386 Th0.016 Mn0.011 Al1.881 Fe2+0.677 Ti0.014 Mg0.042 Si3.568 O12 (OH) -0.019 Na replaced Ca \\
\hline \multirow{9}{*}{ PCS 1-8 } & 1/1. Ca1.306 Ce *0.647 Th0.003 Mn0.024 Al1.98 Fe2+1.004 Ti0.012 Mg0.05 Si3.023 O12 (OH) -0.005 Na replaced Ca \\
\hline & 10/1. Ca1.897 Ce *0.158 Th0.001 Mn0.019 Al2.222 Fe2+0.852 Ti0.008 Mg0.012 Si3.063 O12 (OH) $-0.003 \mathrm{Na}$ replaced Ca \\
\hline & 2/1. Ca1.806 Ce *0.248 Th0.002 Mn0.014 Al2.197 Fe2+0.879 Ti0.011 Mg0.019 Si3.043 O12 (OH) -0.002 Na replaced Ca \\
\hline & 4/1. Ca1.738 Ce *0.321 Th0.001 Mn0.018 Al2.126 Fe2+0.918 Ti0.013 Mg0.036 Si3.043 O12 (OH) $-0.003 \mathrm{Na}$ replaced Ca \\
\hline & 5/1. Ca1.847 Ce *0.229 Th0.001 Mn0.021 Al2.172 Fe2+0.893 Ti0.013 Mg0.023 Si3.04 O12 (OH) \\
\hline & 6/1. Ca1.404 Ce *0.518 Th0.001 Mn0.02 Al2.099 Fe2+0.892 Ti0.011 Mg0.045 Si3.09 O12 (OH) -0.007 Na replaced Ca \\
\hline & 7/1. Ca1.499 Ce *0.557 Th0.001 Mn0.015 Al2.065 Fe2+0.98 Ti0.011 Mg0.038 Si2.998 O12 (OH) $-0.007 \mathrm{Na}$ replaced Ca \\
\hline & 8/1. Ca1.799 Ce *0.223 Th0.001 Mn0.014 Al2.209 Fe2+0.855 Ti0.009 Mg0.017 Si3.071 O12 (OH) -0.003 Na replaced Ca \\
\hline & 9/1. Ca1.739 Ce *0.311 Th0.002 Mn0.011 Al2.203 Fe2+0.866 Ti0.01 Mg0.031 Si3.024 O12 (OH) -0.002 Na replaced Ca \\
\hline $55 \mathrm{~B}-8$ & 2/1. Ca1.155 Ce *0.925 Th0.036 Mn0.021 Al1.818 Fe2+0.754 Ti0.022 Mg0.038 Si3.127 O12 (OH) -0.078 Na replaced Ca \\
\hline \multirow{3}{*}{$20-8$} & 1/1. Ca1.309 Ce *0.619 Th0.029 Mn0.029 Al2.008 Fe2+1.00 Ti0.017 Mg0.023 Si3.051 O12 (OH) -0.002 Na replaced Ca \\
\hline & 2/1. Ca1.09 Ce *0.938 Th0.019 Mn0.036 Al1.796 Fe2+1.186 Ti0.022 Mg0.026 Si3.009 O12 (OH) -0.004 Na replaced Ca \\
\hline & 5/1. Ca1.483 Ce *0.415 Th0.042 Mn0.037 Al2.128 Fe2+0.904 Ti0.012 Mg0.009 Si3.065 O12 (OH) -0.005 Na replaced Ca \\
\hline \multirow{2}{*}{ PCS 2-8 } & 1/1. Ca1.808Ce*0.204 Th0.001 Mn0.015 Al2.193 Fe2+0.845 Ti0.006 Mg0.02 Si3.097 O12 (OH) -0.003 Na replaced Ca \\
\hline & 2/1. Ca1.637Ce*0.263 Th0.003 Mn0.015 Al2.106 Fe2+0.853 Ti0.015 Mg0.028 Si3.17 O12 (OH) $-0.031 \mathrm{Na}$ replaced Ca \\
\hline
\end{tabular}

Ce* is $\mathrm{Ce}+$ Total REE in allanite

\section{REE Settings for Epma}

REE phase analyses were performed using a CAMECA SX-100 electron microprobe analyser at above mentioned laboratory of GSI. The EMP is equipped with five wavelength dispersive spectrometers (WDS), e.g., WDS 1 (TAP crystal), 2 (PET crystal) and 4 (LIF crystal) built-in with low-pressure detectors, and WDS 3 (LPET crystal) and WDS 5 (LIF) built-in with high-pressure detectors (all detectors use P-10 gas). Polypropylene separation windows are used with WDS 1, 2 and 4 and Mylar windows are used with WDS 3 and 5. Calibration, overlap correction and quantifications are performed with the CAMECA SX- 100 Peak Sight-Geo Quanta software package. Pulse-height analysers (PHAs) are adjusted by selecting the differential auto mode for all the elements. Matrix effects were eliminated by using the X-PHI correction method proposed by Merlet $(1992,1994)$. REE phase are analysed using $20 \mathrm{kV}$ acceleration voltage and $20 \mathrm{nA}$ beam current with $1 \mu \mathrm{m}$ size beam. The signals used are $\mathrm{Si} \mathrm{Ka}, \mathrm{Ca} \mathrm{Ka}, \mathrm{Na} \mathrm{Ka}, \mathrm{P} \mathrm{Ka}, \mathrm{Al} \mathrm{Ka}$, Ti Ka, Fe Ka, Y La, La La, Ce La, Pr Lb, Nd Lb, Sm La, Eu La, Gd La, Tb La, Dy Lb, Ho La, Er La, Tm La, Yb La, Lu La, Pb Ma, Th Ma, U $\mathrm{Mb}, \mathrm{Nb} \mathrm{La}$, Ta La for calibration of elements using natural standards for $\mathrm{Si}, \mathrm{Ca}, \mathrm{P}, \mathrm{Fe}, \mathrm{Pb}$, synthetic standards for Al, Ti, REE, Y, U, Th. Standards used are wollastonite for $\mathrm{Si}, \mathrm{Ca}$, apatite for $\mathrm{P}$, corundum for $\mathrm{Al}$, haematite for $\mathrm{Fe}$, albite for $\mathrm{Na}, \mathrm{Pb}$ on crocoite, synthetic glass standards for REE, $\mathrm{U}$, Th and $100 \%$ metal standards for $\mathrm{Nb}$, Ta. The counting time for peak measurement is $10 \mathrm{~s}$ and with half of the peak measurement time allotted for background measurement.

\section{Petrography}

Migmatite gneiss (protolith age 2.75-2.698 Ga; Dharma Rao et al.,
2011) essentially consists of quartz, plagioclase, and orthoclase and less amount of microcline as major minerals, having biotite, sodic hornblende and garnet (at places) as subordinate or minor minerals. Allanite, epidote, sphene, magnetite, ilmenite and zircon are the accessory minerals in migmatite gneiss. Allanite occurs as discrete, primary, euhedral crystals overprinting and pushing aside the major foliation $\left(\mathrm{S}_{2}\right)$ defined by biotite-amphibole and quartzofeldpathic minerals, thereby suggesting that it formed as a result of reaction between a REE bearing fluid rich in $\mathrm{P}$ and $\mathrm{F}$ and the silicate minerals (Fig. 2, 4) (see, Arden and Halden, 1999). The proportion of plagioclase and orthoclase is varying regionally and perthitic texture is very common in migmatite gneiss. Some cluster of mafic minerals like sodic hornblende and biotite is associated with mafic bands of migmatite gneiss. Biotite, represented by $\mathrm{Fe}-\mathrm{Mg}$ rich annite, in migmatite gneisses are seen to form by breakdown of muscovite + quartz assemblage above the granite melting curve. The migmatite gneiss serves as host for the intrusive Amet granite and the monzonite-syenite-alkali syenite rocks.

Petrographically, Amet Granite (1870 \pm 200 Ma; Chaudhary et al., 1984 ) is mainly composed of plagioclase, quartz, and alkali feldspars as the major constituent and hornblende with minor amount of biotite as minor constituents and sphene, epidote, allanite and magnetite as accessory minerals. Garnet porphyroblast is also present within the Amet granite. Both primary and secondary biotite is present within the Amet granite. The primary biotite is Annite compositionally and is aligned along the foliation plane and secondary biotite is haphazardly oriented. The secondary biotite is formed due to the metamorphism of amphiboles.

Syenite-alkali feldspar syenite is predominantly composed of alkali feldspar with small amount of plagioclase and more than 5\% quartz, whereas monzonite is predominantly composed of alkali feldspar and 


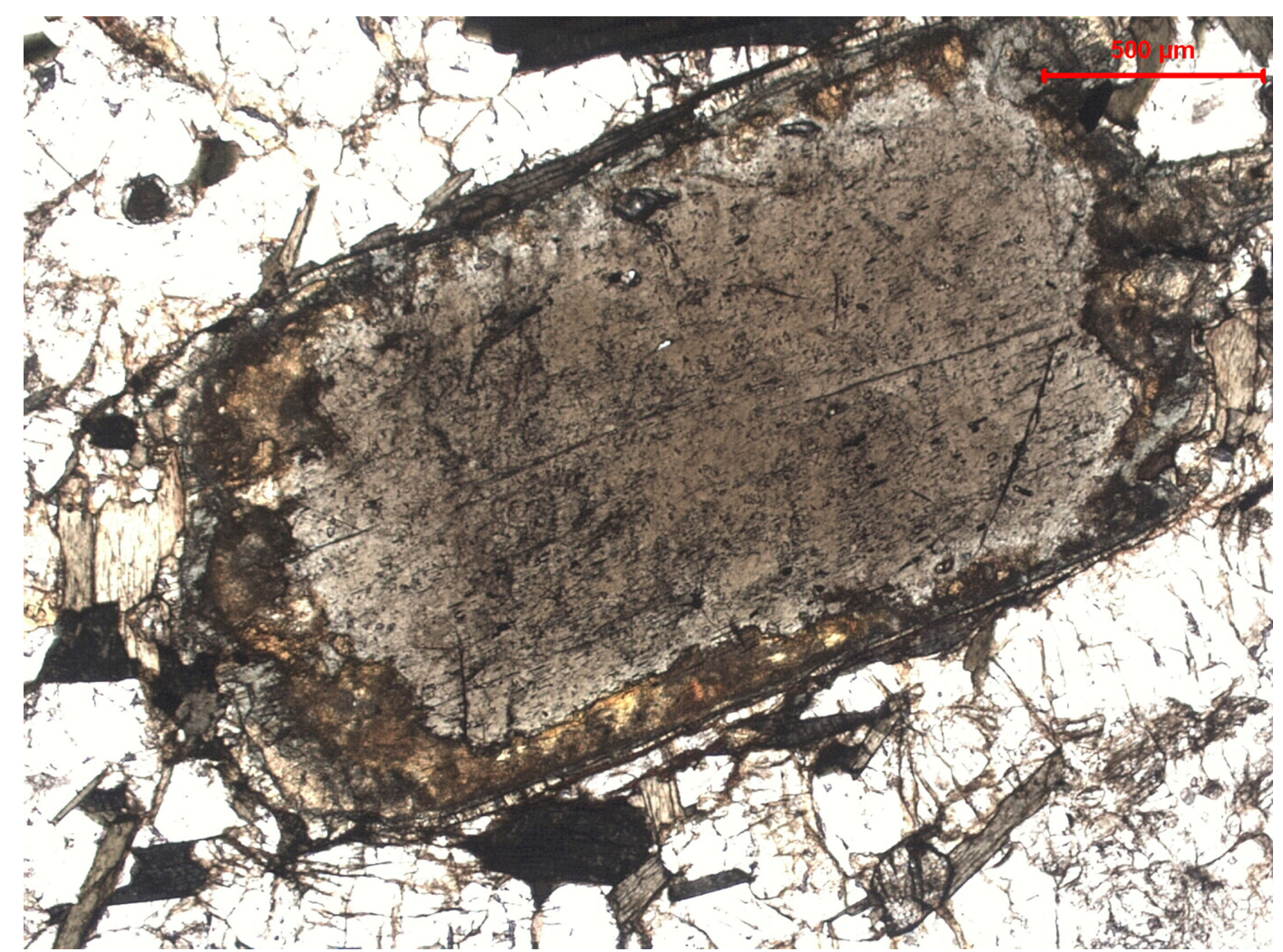

Figure 2. 35-8-borana allanite with 1 set cleavage parallel to 100 or 010 suggesting the mineral is not altered (Molloy, 1959). Note rim of britholite (darker brown) around allanite core (lighter brown).

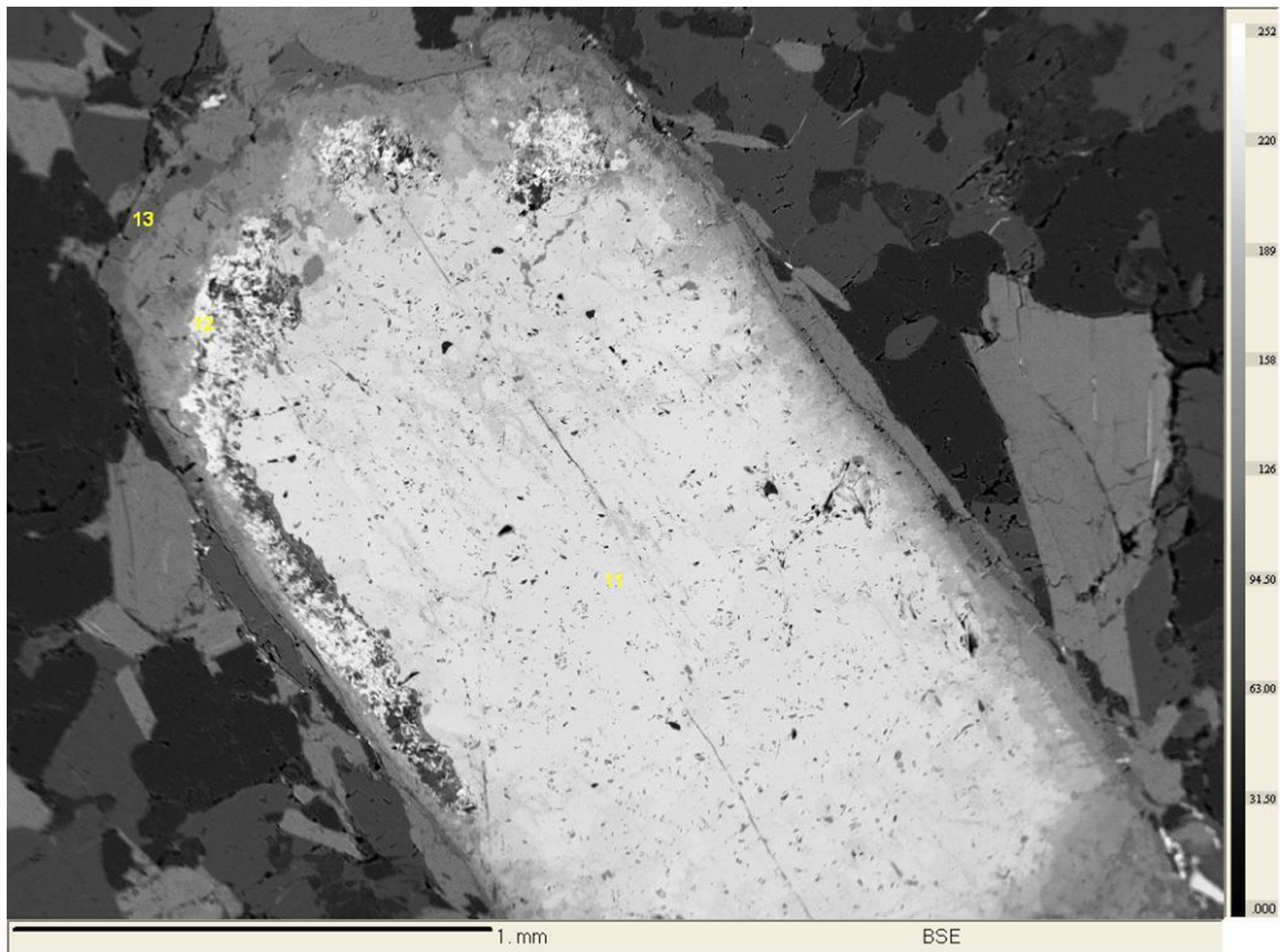

Figure 3. BSE image of same grain (35-8) with point 11-Allanite core, 12-Allanite rim, 13- outer rim Allanite (alteration). 


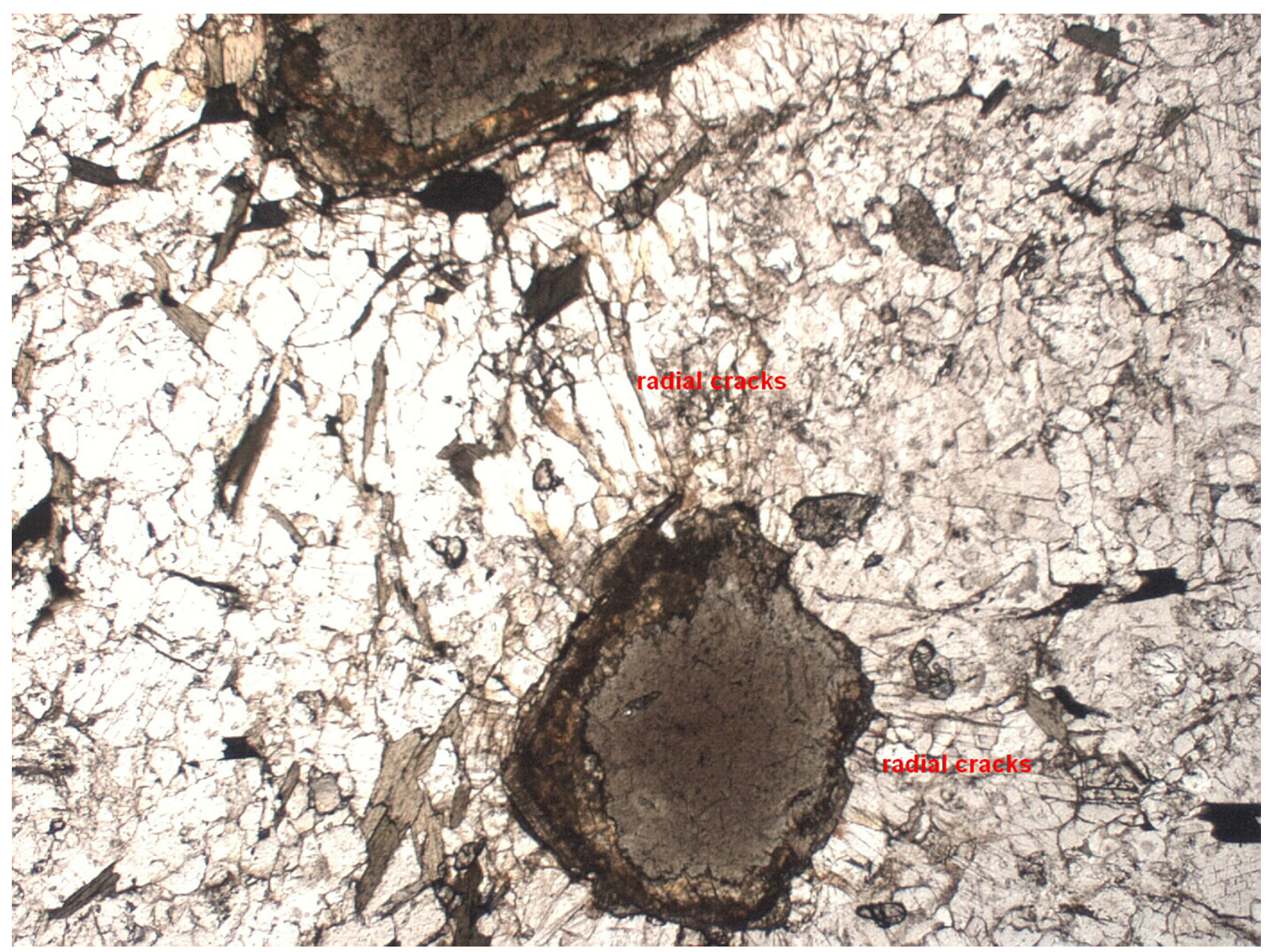

Figure 4. Allanite grain with radial expansion cracks. PPL X10.

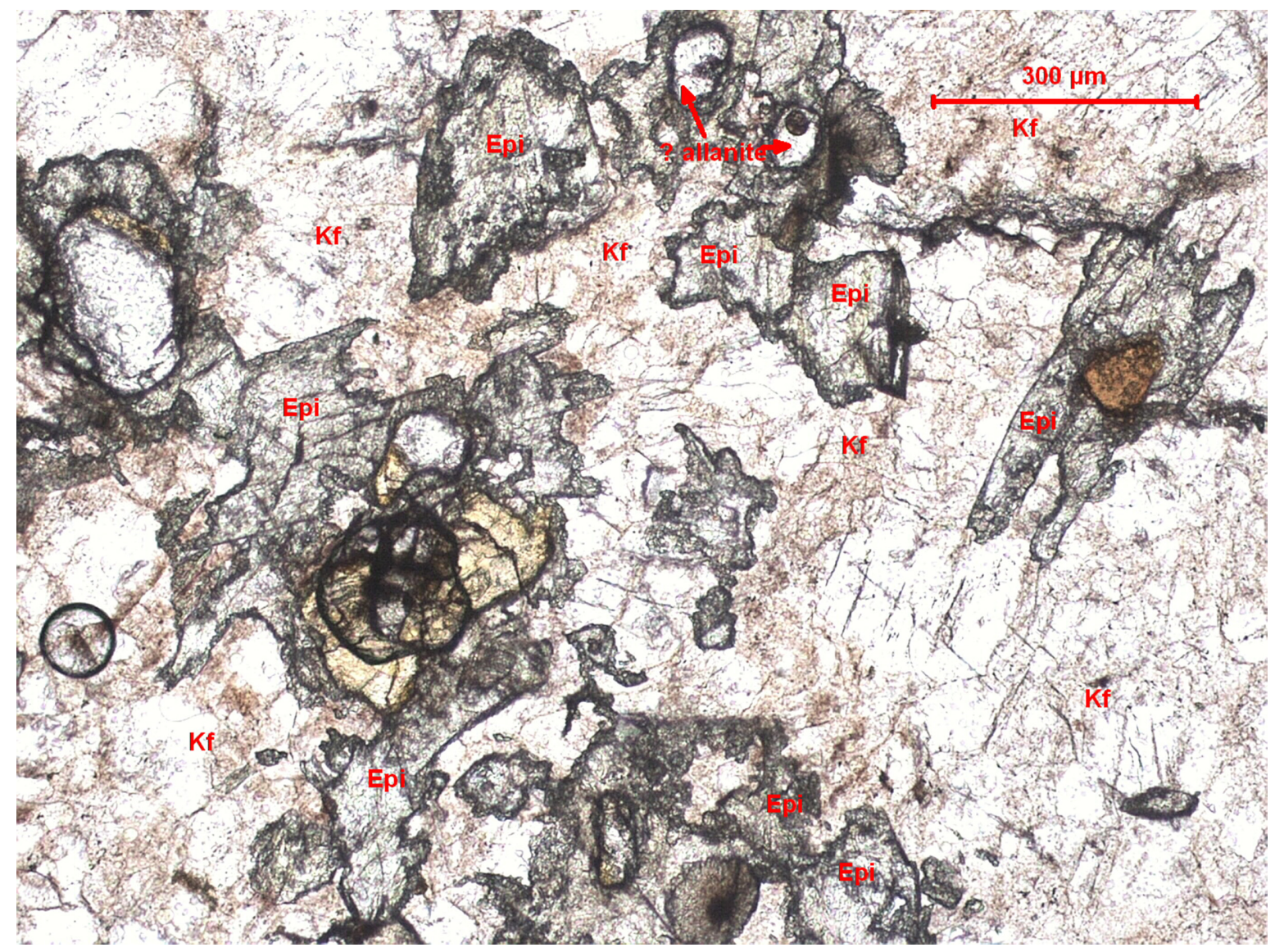

Figure 5. Sample 20-8 (syenite) Allanite-epidote in a groundmass of k-feldspar. Allanite rimmed by britholite or thorite; centre of epidote (Epi) is having glass appearance (thorite). One big allanite on left side. PPL X 10. 


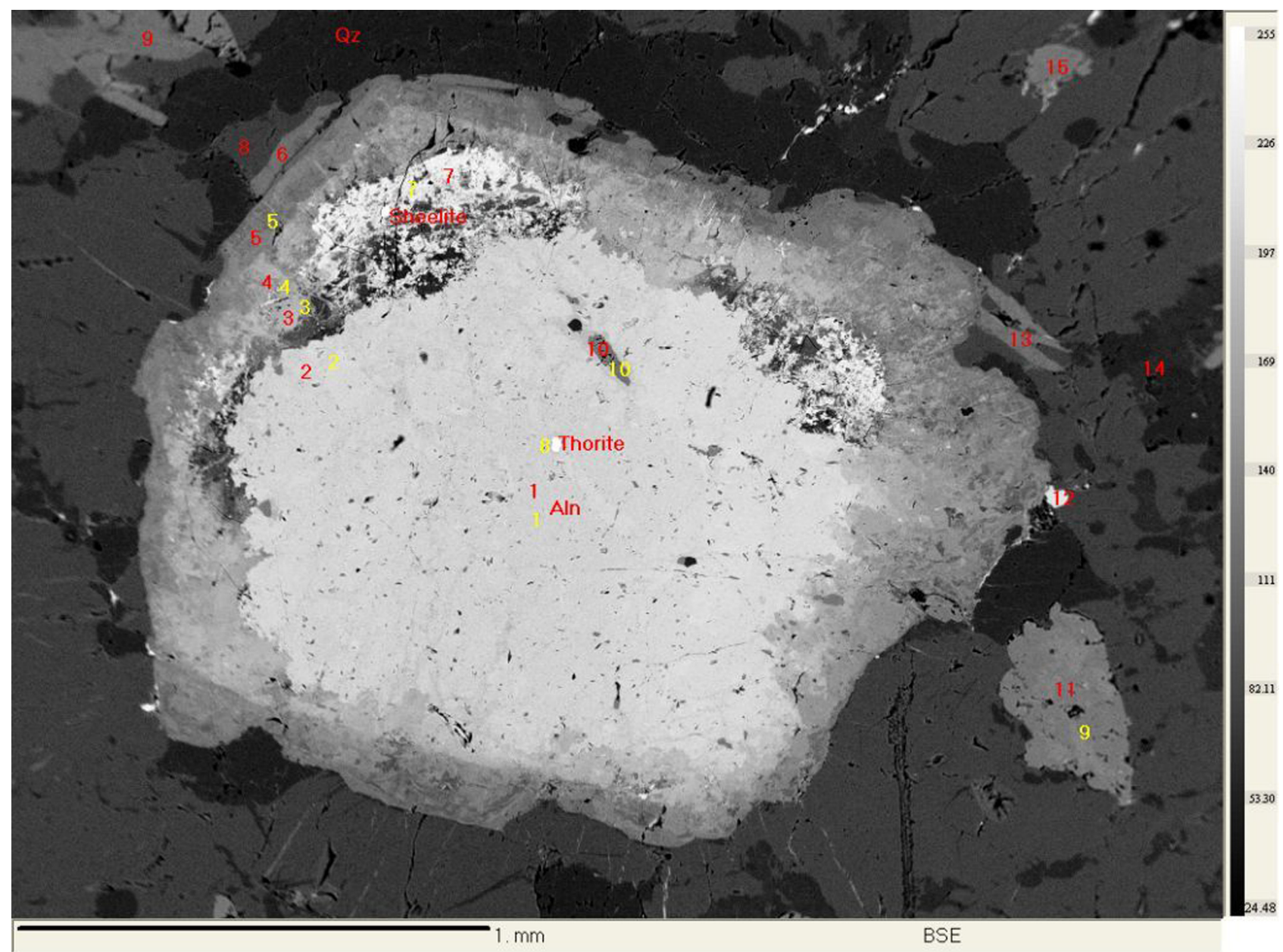

Figure 6. BSE image of sample 35-8. Core portion is allanite, britholite (white part) with a white grain of scheelite at NW and NE parts, \& thorite in centreas alteration. Points 2-rim of allanite, 3-alteration part, 4-REE mineral, 5 \& 6-alteration part, 7-britholite with scheelite, 8$\mathrm{Kf}$, 9-Bi, 10, 11, 15-titanite, 12-iron phase, 13-Bi. (yellow points are REE analysed).

plagioclase with less than $5 \%$ quartz. Action of fluid is noticeable as foliation parallel replacement of Amet granite by monzonite is seen which may be due to magma mixing (Fig. 1c). The ages of these intruded rocks (Syenite/alkali feldspar syenite and monzonite) are not yet known but logically they are supposed to be little younger in age than the Amet Granite as it intruded and replaced the granite.

Allanite occurs both as discrete crystals as well as irregular segregations, whereas britholite occurs only as irregular masses forming rims in allanite crystals. The allanite with its britholite rim, typically share sharp contacts with host silicate minerals k-feldspar, plagioclase, biotite, sodic amphibole (Fig. 2, 3 and 8). BSE images of allanite show both normal growth induced magmatic zoning and complicated internal zoning consisting of patchwork of variable domains in brightness (Fig. 3, 6 and 7). Petrography also reveals presence of zoned allanite euhedral crystals (Fig. 2 and 4) with peripheral rims of britholite and titanite and core of allanite. Radial cracks developed not due to radioactive decay, but these are expansion cracks due to alteration from allanite to britholite-thorite (Fig. 4). Apatite, zircon, titanite, britholite, thorite, U-bearing minerals are identified through EPMA studies (Fig. 8). Apatite is seen to be enclosed in allanite (Fig. 8). Epidote changing to allanite rimmed by britholite is seen in Fig. 5 wherein big grains of allanite are forming in the core of epidote.

\section{Geochemistry and Mineralogy}

\section{Geochemical Characterisation}

The whole rock analysis has been given in Table 1 and 2. The TAS diagram (Fig. 9, Cox et al., 1979) shows that the migmatite gneiss falls in granitic field whereas syenite and amphibolite fall in their respective fields and a crystallisation trend from migmatite gneissAmet granite-syenite is observed.

The chemical analyses of the migmatite gneiss indicate that they all have more than $66 \% \mathrm{SiO}_{2}$ and hence classified as acidic suite (Carmichael et al., 1974). Their mean $\mathrm{Al}_{2} \mathrm{O}_{3}$ concentration is $14.0 \%$ classifying them as low- $\mathrm{Al}$ gneisses (Monrad, 1983). Their $\mathrm{Al}_{2} \mathrm{O}_{3} / \mathrm{Na}_{2} \mathrm{O}+\mathrm{K}_{2} \mathrm{O}$ ratio is less than 3.4 pointing to their sub-alkaline nature (Bose, 1989). A/CNKA/NK diagram (Fig. 10) of Shand (1943) also discriminate these rocks as metaluminous. In the $\mathrm{Na}_{2} \mathrm{O} \mathrm{Vs} \mathrm{K}_{2} \mathrm{O}$ diagram (Fig. 11) of Smith (1982), all our samples of Amet granite plot in the field of S-type granites. In A/CNK-A/NK diagram of Shand (1943) all the samples of Amet granite fall in Metaluminous field (Fig. 10).

The chemistry of the migmatite gneiss indicates a calc alkaline nature and the syenite-alkali feldspar syenite intrusives having alkaline nature, 


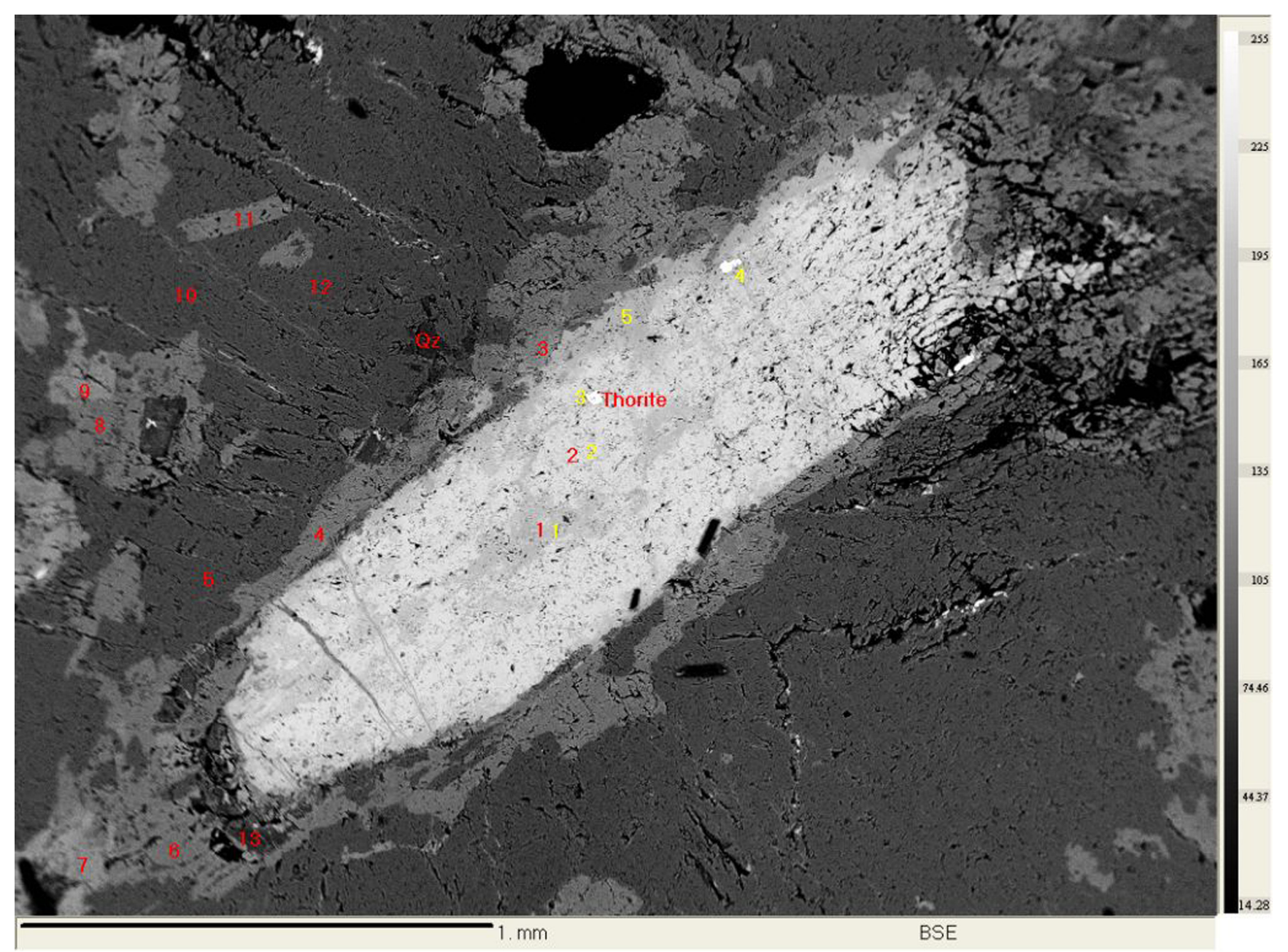

Figure 7. Sample 20-8 BSE image showing points (Yellow) 1\& 2-allanite core, 3 \& 4-thorite, 5-allanite rim.

thereby indicating a genetic correlation between the migmatite gneiss and the alkaline intrusives. In the Na-K-Ca triangular diagram after Barker and Arth, (1976), these rocks show trondhjemitic characteristics (Fig. 12). These rocks are metaluminous when plotted in Shand's diagram (Shand, 1943).

$\mathrm{Zr}$ and $\mathrm{Th}$ is moderate to high in migmatite gneiss, Amet Granite and alkali intrusives. $\Sigma$ REE value (Table 2) is low to moderate in migmatite gneiss (156.12 to $627.40 \mathrm{ppm}$ ) and high in syenite-alkali feldspar syenite (1410.29 to $2071.20 \mathrm{ppm}$ ) which may indicate that the carrier of the REE mineral allanite-britholite is alkaline intrusive syenite-alkali feldspar syenite which deposited their REE content in migmatite gneiss along and across foliations and micro shear (Fig. 2 and 13). The Zr, Y, La, Ce values increase with release of silica in the system, whereas values of $\mathrm{Rb}, \mathrm{Sr}, \mathrm{Ni}, \mathrm{Cr}, \mathrm{Ba}$, decrease from syenitealkali feldspar syenite to Amet granite and migmatite with increase of silica (Table 1 and 2). This is consistent with crystallisation of mafic phases in early stages of magmatic crystallisation. The feldspars of these rocks when plotted in Ab-An-Or diagram (Fig. 14) show feldspars of monzonite as of albite composition and alkali feldspar syenite of orthoclase composition. The mineral composition of biotite of Amet Granite and migmatite gneiss falls in the field of annite thereby indicating higher $\mathrm{X}_{\mathrm{Fe}}$ content (Fig. 15).

\section{Mineral Chemistry}

Total silicate and REE values and of five samples containing allanite, analysed by EPMA, are given in Table 3. The analysis indicates the presence of mineral phases as allanite, britholite, thorite, titanite, apatite, zircon, as major phases and minor scheelite and Uranium-bearing phase in the samples. The cation proportion of the allanite calculated for these five samples is given in Table 4 and the mineral formula of allanite calculated on the basis of $13(\mathrm{O}, \mathrm{OH})$ is given in Table 5. The composition of allanite ranges from $\mathrm{Ca}_{1.031 \mathrm{Ce}} \mathrm{e}_{0.971} \mathrm{Th}_{0.034} \mathrm{Mn}_{0.039} \mathrm{Al}_{1.525}$ $\mathrm{Fe}^{2+}{ }_{1.316} \mathrm{Ti}_{0.97} \mathrm{Mg}_{0.085} \mathrm{Si}_{3.007} \mathrm{O}_{12}(\mathrm{OH})$ to $\mathrm{Ca}_{1.897} \mathrm{Ce}^{*}{ }_{0.158} \mathrm{Th}_{0.001} \mathrm{Mn}_{0.019} \mathrm{Al}_{2.222}$ $\mathrm{Fe}^{2+}{ }_{0.852} \mathrm{Ti}_{0.008} \mathrm{Mg}_{0.012} \mathrm{Si}_{3.063} \mathrm{O}_{12}(\mathrm{OH})$, where $\mathrm{Ce}{ }^{*}$ is $\mathrm{Ce}+$ total of other REE values. The mineral phase identified, thus, is allanite (Ce), the allanite phases being $\mathrm{CaO}$ and $\mathrm{Ce}_{2} \mathrm{O}_{3}$ rich (see Table 1). Silica and alumina show positive correlation in allanite. As the silica proportions decreases and total REE increases (Table 3), allanite changes to britholite (sample 35-8, 55B-8) and also thorite (sample 20-8). Therefore, with release of $\mathrm{SiO}_{2}$, total REE increases. Some grains of scheelite and uranium mineral phase are also identifiable.

From thin section study it is seen that allanite is growing over epidote and other silicate minerals (Fig. 5). More total wt\% of EPMA data may be related to interference of some elements in allanite because of 


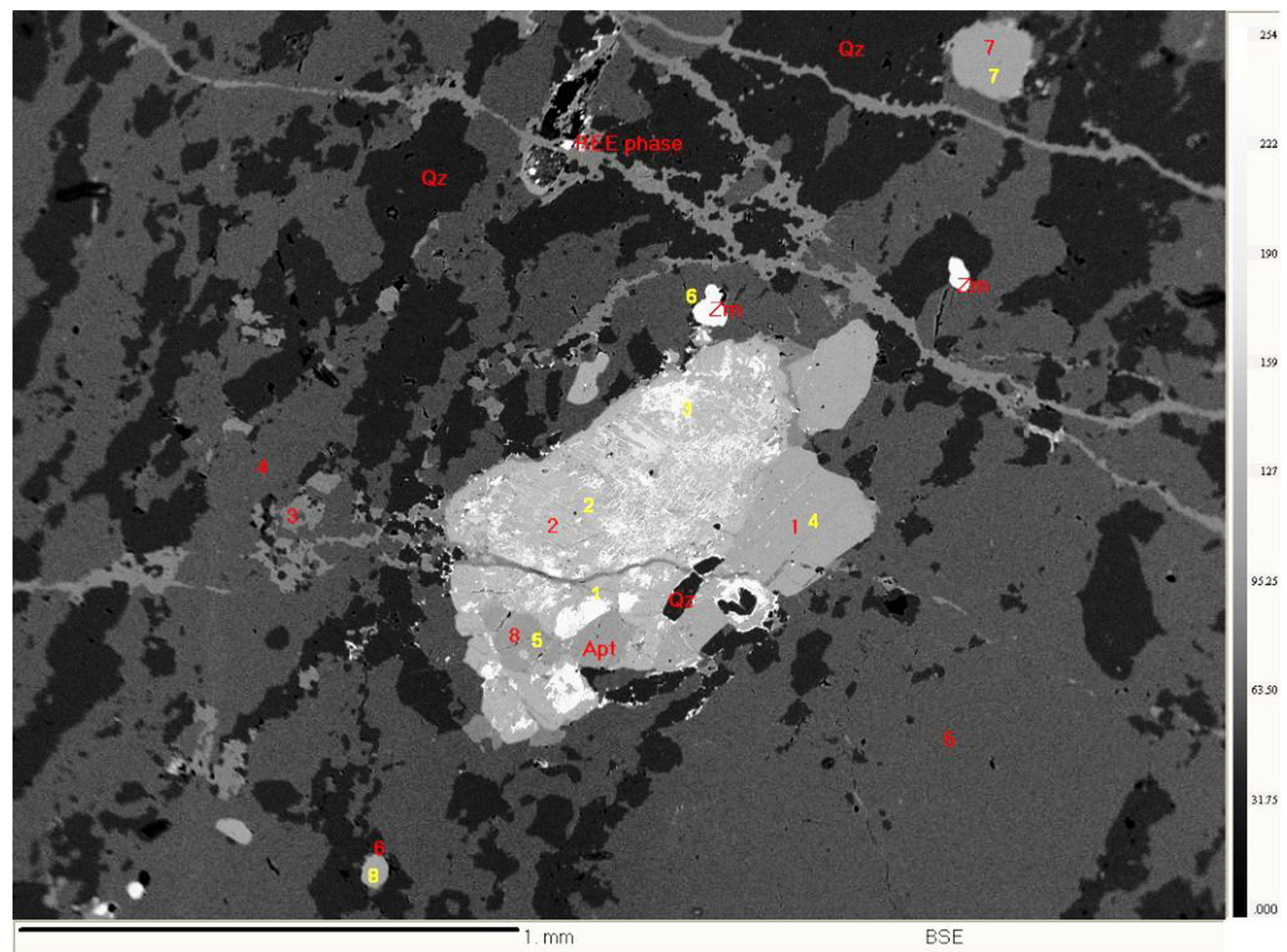

Figure 8. Sample 55B-8-BSE image showing points (yellow) 1, 2 \& 3-allanite (REE rich white part), 4-titanite, 5-apatite in allanite, 6-zircon, 7-apatite, 8-titanite.

its development from fluid action on epidote thereby crystallising allanite and other REE minerals. Core part of allanite changes to britholite as white rim (Fig. 6) which is REE rich britholite (Ce rich, Table 3), an alteration of allanite. Decrease in $\mathrm{MnO}$ is also related to increase in $\mathrm{La}$ and Ce making alteration of allanite to britholite. In one sample PCS $1 / 8, \mathrm{FeO}$ is $1.09 \%, \mathrm{SiO}_{2} 29.55 \%$, but britholite could not form as $\mathrm{SiO}_{2}$ did not move out of the system. As the percentage of $\mathrm{FeO}$ increases, different rims of REE minerals are formed as evident from petrographic and EPMA studies (Fig. 2, 3, 4, and 6). $\mathrm{FeO}$ is more in allanite than britholite and thorite in this case (sample 35/8, Table 3). Increase in total REE and with release of $\mathrm{SiO}_{2}$ from system, allanite changed to britholite and thorite indicating role of fluid in this case. Another important observation is that Ca+LREE replaces Mn+HREE (Fig. 16). As HREE (Gd+Dy) increases, Mn also increases (Fig. 17); and as $\mathrm{Ca}$ increases, $\mathrm{Mn}$ decreases (Fig. 18) indicating a direct replaceable relation between $\mathrm{Ca}, \mathrm{Mn}, \mathrm{HREE}$ and LREE. The chondrite normalised pattern of $\Sigma$ REE in allanite vis-a-vis the chondrite normalised pattern of $\Sigma \mathrm{REE}$ in migmatite gneiss containing these allanite are compared in Fig. 20 which shows a compatible relation of $\Sigma$ REE of rock vis-avis the mineral allanite contained within it, indicating primary allanite. Migmatite host shows a flat pattern with significant negative Eu anomaly (Fig. 20) as is expected in Achaean-Proterozoic rocks, and is explained as original source character of magma (Sun and Nesbitt, 1978). Since allanite crystallised in later period of magmatic crystallisation, when volatile rich fluid containing REE, rare earth metal and many such ore minerals of $\mathrm{U}$, Ti of significance crystallise; the compositional variation in allanite REE and host REE is imminent. This observation, therefore, indicates primary nature of allanite of study area.

The $\mathrm{Al}_{2} \mathrm{O}_{3}-\mathrm{Fe}_{2} \mathrm{O}_{3}$ - $\mathrm{REE}$ diagram (Fig. 19) indicates that the allanite of study area are falling in intracontinental field, which indicates crystallisation of allanite in consonant with the tectonic setting of migmatite gneisses and granites of the Rajasthan craton (See Hoshino et al., 2005).

\section{Discussion}

Magma genesis and assimilation (mixing) of deeper crustal rocks is a possible hypothesis, but mingling or comingling of magma is more plausible in this case (Kumar, 2014) as the migmatite gneiss which is a granitic gneiss, is trondhjemitic in nature (Fig. 12); is replaced or intruded by pulses of syenite-alkali feldspar syenite rocks containing epidote dikes and veins, with thoroughly dispersed primary allanite grains in the rocks. The age of migmatite gneiss is $2.75-2.698 \mathrm{Ga}$ 
TAS (Cox et al., 1979)

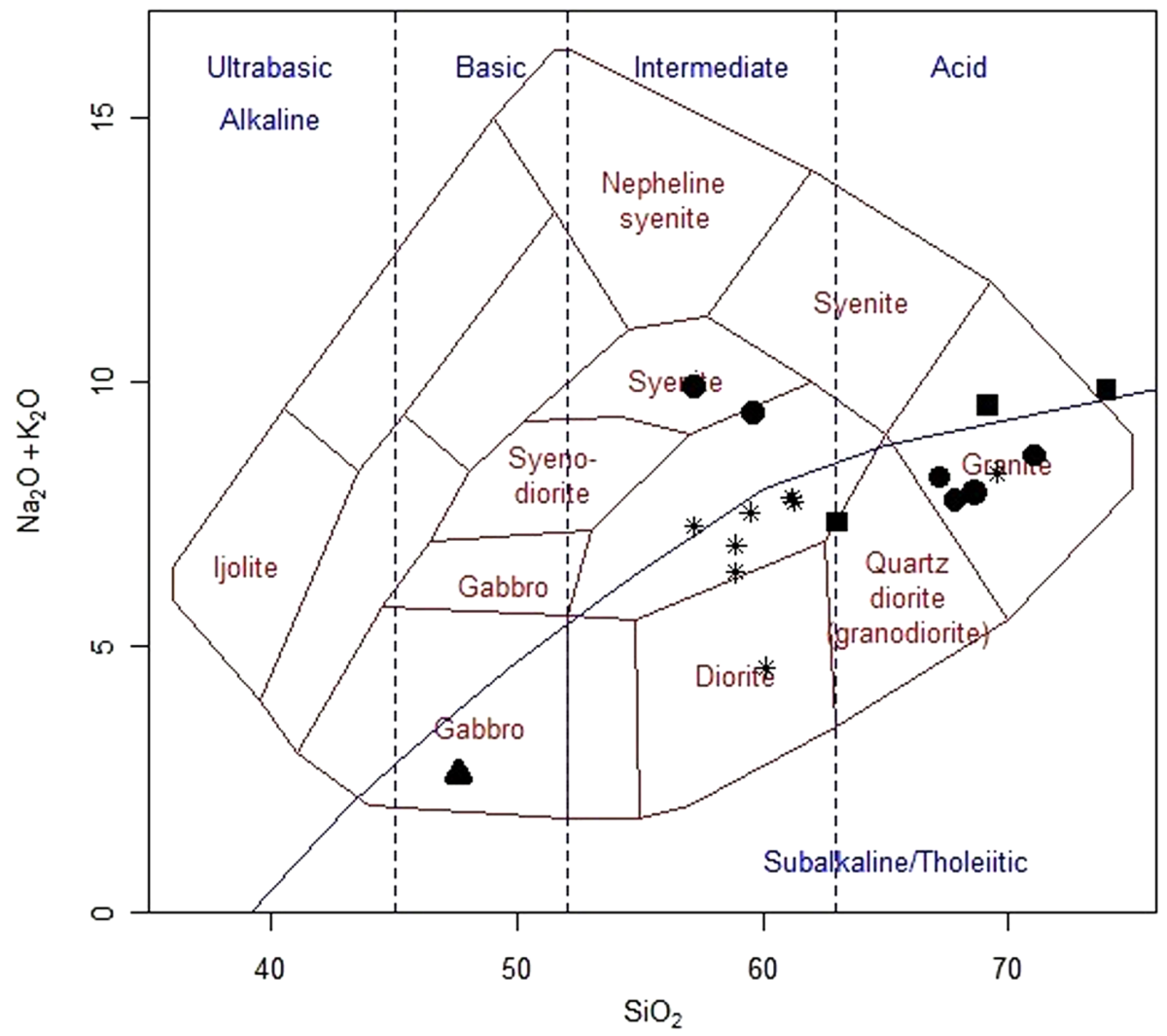

Figure 9. TAS diagram of syenite (asterisk), Amet Granite (filled circle), Migmatite (filled square), Amphibolite (triangle) after Cox et al. (1979).

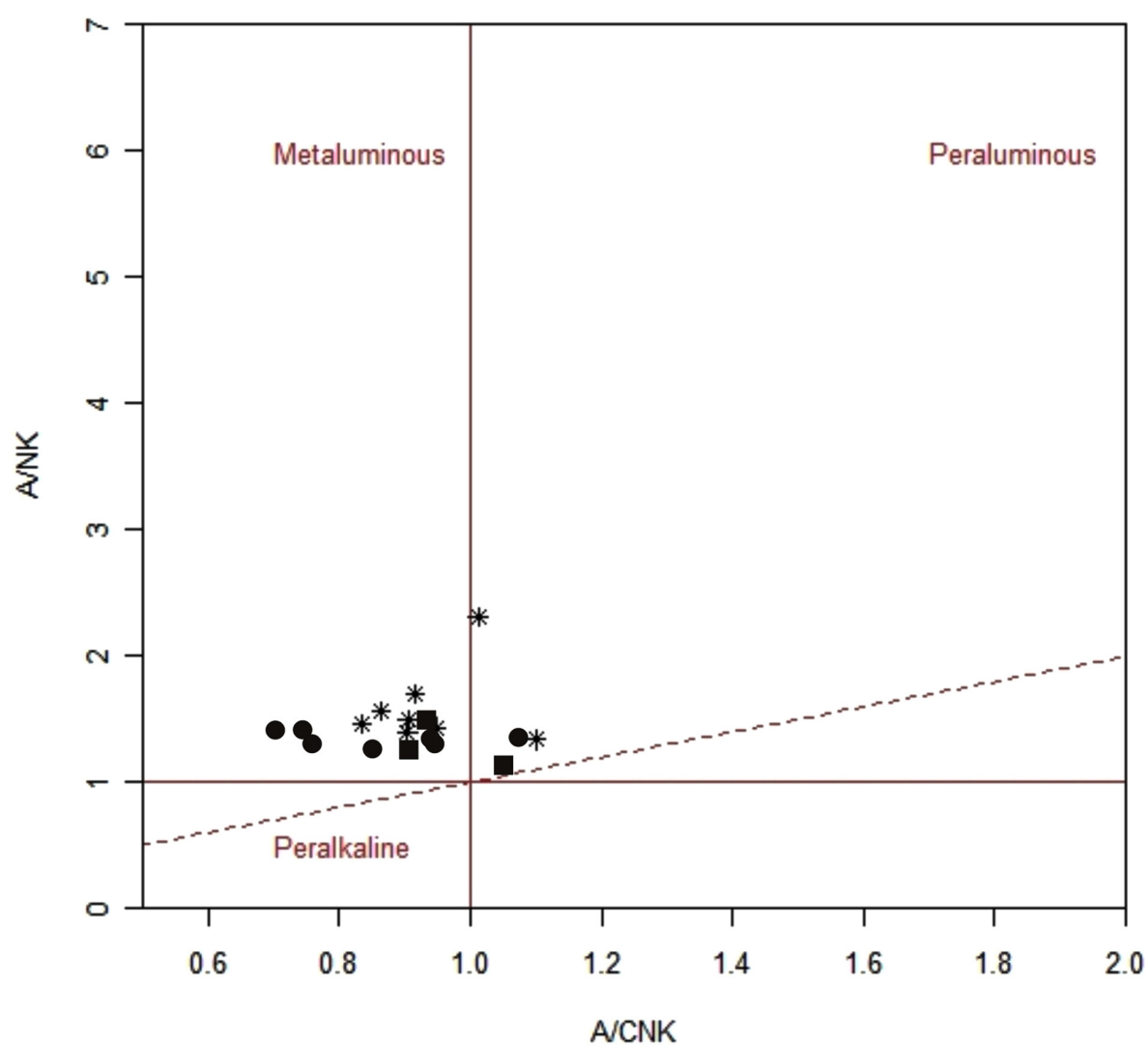

Figure 10. A/CNK vs A/NK diagram after Shand (1943). Symbols as in Fig. 9. whereas the syntectonically emplaced amphibolite within it is $2.83 \pm 0.05 \mathrm{Ga}$. The intrusive plutonic Amet Granite is $1870 \pm 200$ Ma. Chronologically, migmatite gneiss is the oldest crust which was intruded syntectonically by mafic rocks (now amphibolite), and finally by a melt generated from migmatite gneiss as the Amet Granite pluton. The syenite-alkali feldspar syenite and monzonite dikes intruded last in succession cutting across all rock types in study area.

The minerals epidote and also allanite are seen in the alkaline rocks ubiquitously. The mineral composition of epidote is $\mathrm{Ca}_{2}\left(\mathrm{Al}_{2}, \mathrm{Fe}\right)\left(\mathrm{SiO}_{4}\right)$ $\left(\mathrm{Si}_{2} \mathrm{O}_{7}\right) \mathrm{O}(\mathrm{OH})$ and is an end member of solid solution series with clinozoisite. Members of the epidote mineral group have a crystal structure that consists of isolated and paired silica tetrahedrons. They share a generalized chemical composition of $\mathrm{A}_{2} \mathrm{M}_{3}\left(\mathrm{Si}_{2} \mathrm{O}_{7}\right)\left(\mathrm{SiO}_{4}\right) \mathrm{O}(\mathrm{OH})$. "A" is a pairing of calcium, manganese, strontium, lead, or sometimes a REE. "M" is usually aluminum pairing with iron, magnesium, manganese, or vanadium. Now here in the present case, $\mathrm{Ca}$ is paired with $\mathrm{Ce}$, Th and $\mathrm{Mn}$, and $\mathrm{Al}$ is paired with $\mathrm{Mg}$ and $\mathrm{Fe}$ (Table 5); thereby allanite is formed in profuse in the alkaline rocks during magmatic crystallisation itself. The more the silica released from the system, new species as britholite and thorite have formed indicating a role of fluid in its crystalisation (Mueller and Saxena, 1977). The chondrite normalized REE in migmatite gneiss and allanite mineral is compatible (Fig. 20), thus indicating a primary nature of allanite in the host. Therefore, it is envisaged that the carrier of the REE mineral is alkaline intrusive syenite/alkali feldspar syenite rocks which deposited their REE minerals during ascent of alkaline magma in semi-consolidated state in migmatite gneiss along and across foliations and micro shears, due to reaction between a REE bearing fluid rich in $\mathrm{P}$ and $\mathrm{F}$ brought up by alkaline rocks, and the silicate minerals, mainly biotite and sodic hornblende of the migmatite gneiss (Fig. 2) and Amet granite (Fig. 13) (cf., Arden and Halden, 1999).

Field evidences of syenite-alkali feldspar syenite impregnating migmatite gneiss and amphibolite (syntectonically intruded in migmatite, and pre syenite) as dikes, sills and veins; and the petrographic study indicated that the syenite-alkali feldspar syenite acted as carriers of the REE bearing mineral phases from deep 0 crustal source at higher temperatures. The syenitealkali feldspar syenite magma is derived from deeper crustal source since it is not oxidised as seen petrographically as well as the chemical 


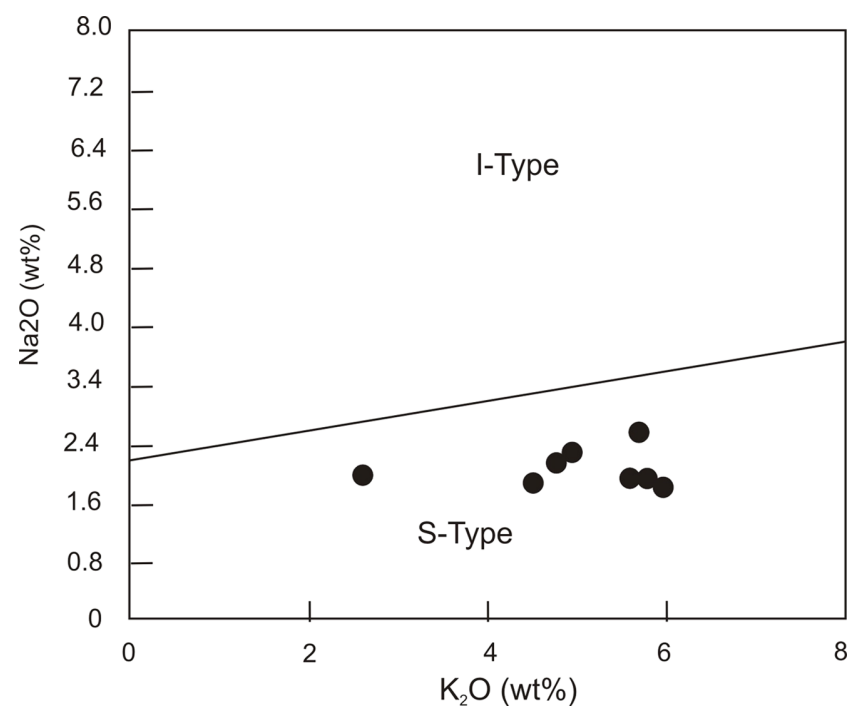

Figure 11. $\mathrm{K}_{2} \mathrm{O} / \mathrm{Na}_{2} \mathrm{O}$ diagram of Amet Granite. Symbols as in Fig. 9.

data indicating negative Eu anomaly. Higher temperature of the fluid or magma is indicated by the fact that the granite melting curve has been crossed in migmatite gneiss through the breakdown reaction of Muscovite + Quartz $\rightarrow \mathrm{Al}_{2} \mathrm{SiO}_{5}+\mathrm{K}$-feldspar + Biotite $+\mathrm{H}_{2} \mathrm{O}$. Absence of primary muscovite and presence of biotite in the migmatite gneiss is an indicator of these phenomena where the emplacement of the

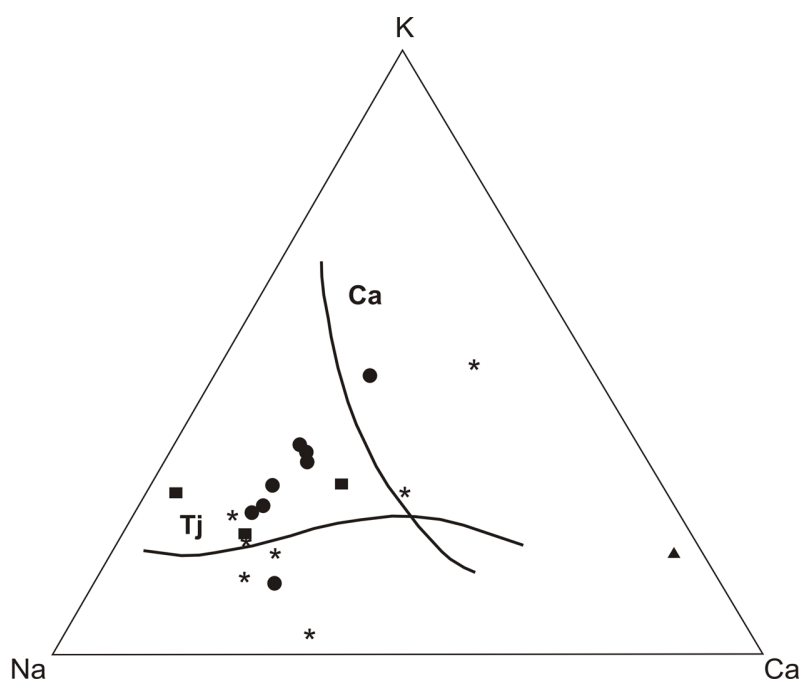

Figure 12. K-Na-Ca diagram of migmatite gneiss, Amet granite, syenite-alkali feldspar syenite and amphibolite. Symbol as in Fig. 9.

syenite-alkali feldspar syenite magma can be considered above the Muscovite + Quartz stability curve (Winkler, 1974). The role of fluid in magmatism and allanite mineral crystallisation is also indicated by reaction between a REE bearing fluid rich in $\mathrm{P}$ and $\mathrm{F}$ brought up by alkaline rocks, and the silicate minerals biotite and sodic hornblende of the migmatite gneiss (Fig. 2) and Amet granite (Fig. 13) (Arden and

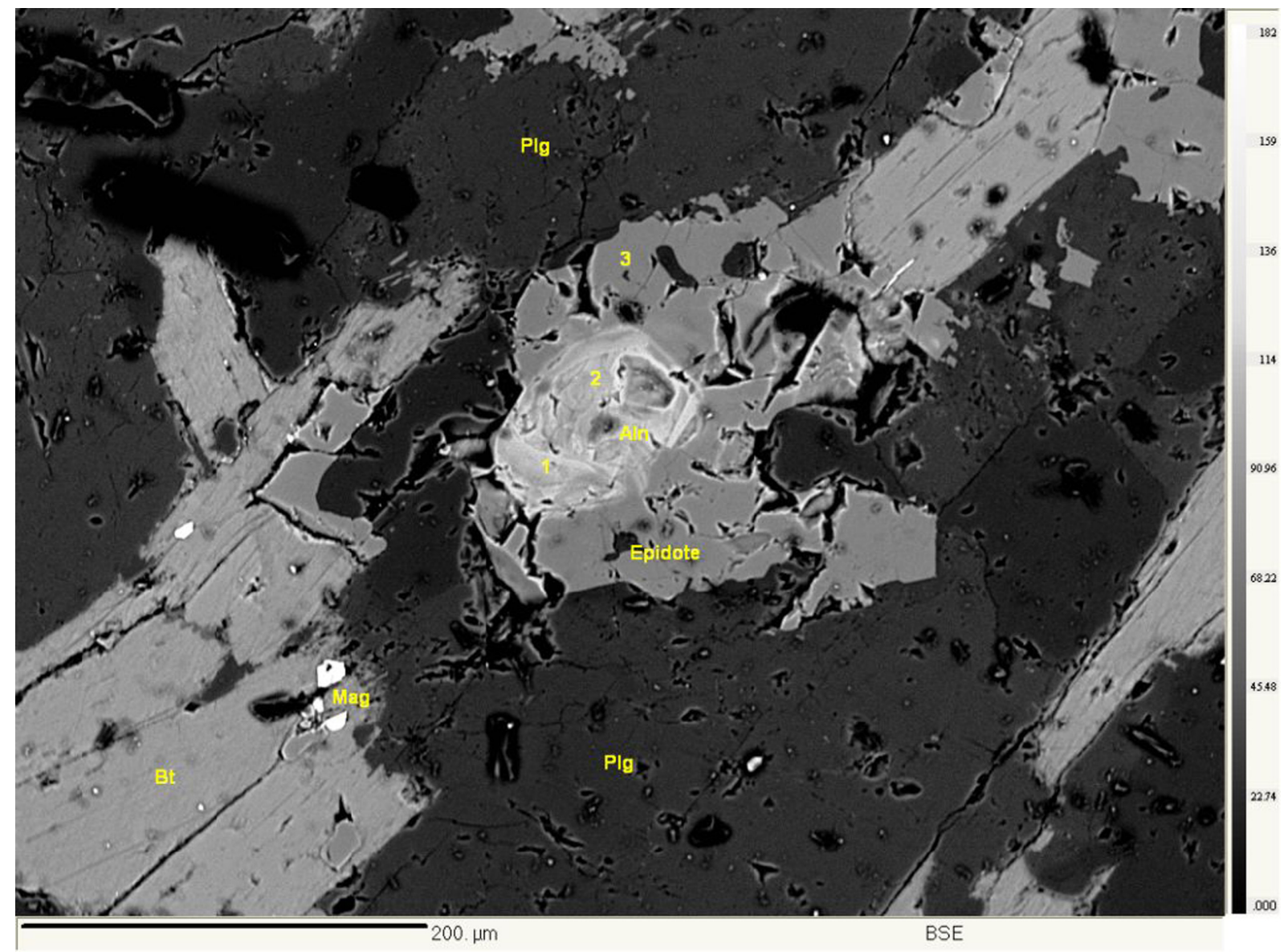

Figure 13. Sample PCS 02-08 BSE image showing epidote containing allanite having reaction relation with biotite and feldpar in groundmass. Aln (allanite), Bt (biotite), Plg (plagioclase). 


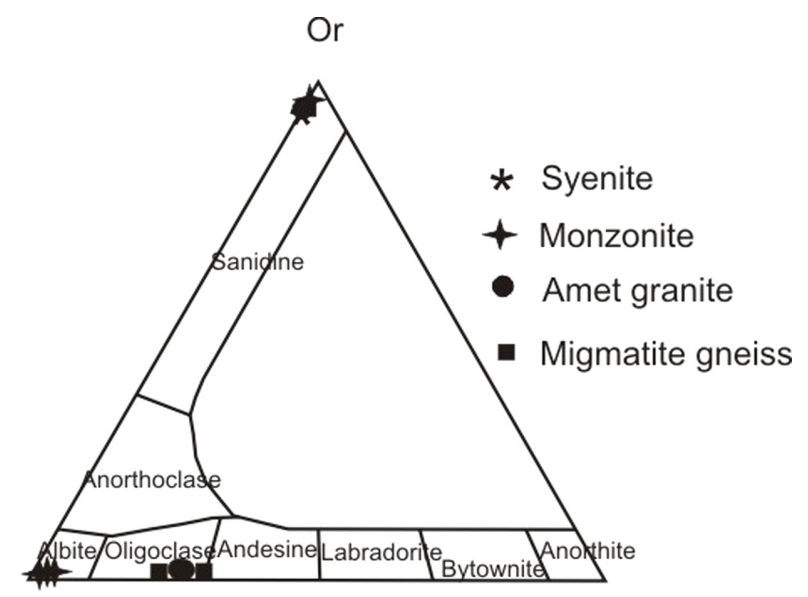

$\mathrm{Ab}$

An

Figure 14. Plot of plagioclase and $k$-feldsar analysis of syenite (asterisk), monzonite (star), Amet Granite (filled circle), Migmatite (filled square).

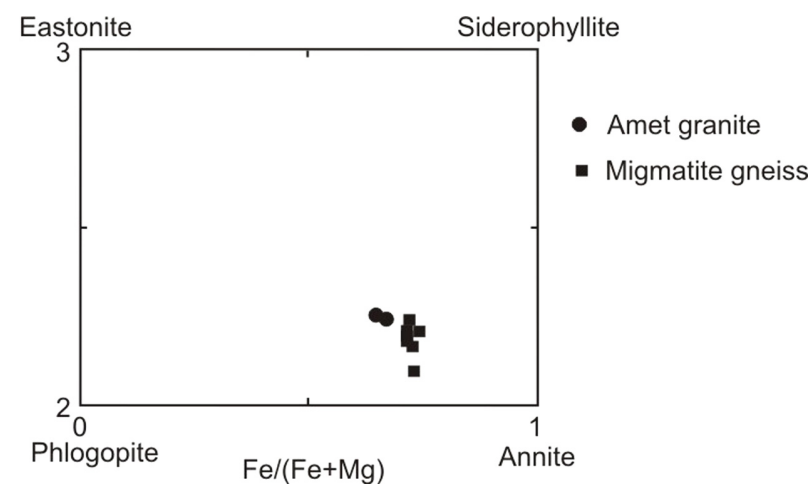

Figure 15. Plot of biotite of Amet Granite (filled circle), Migmatite (filled square).

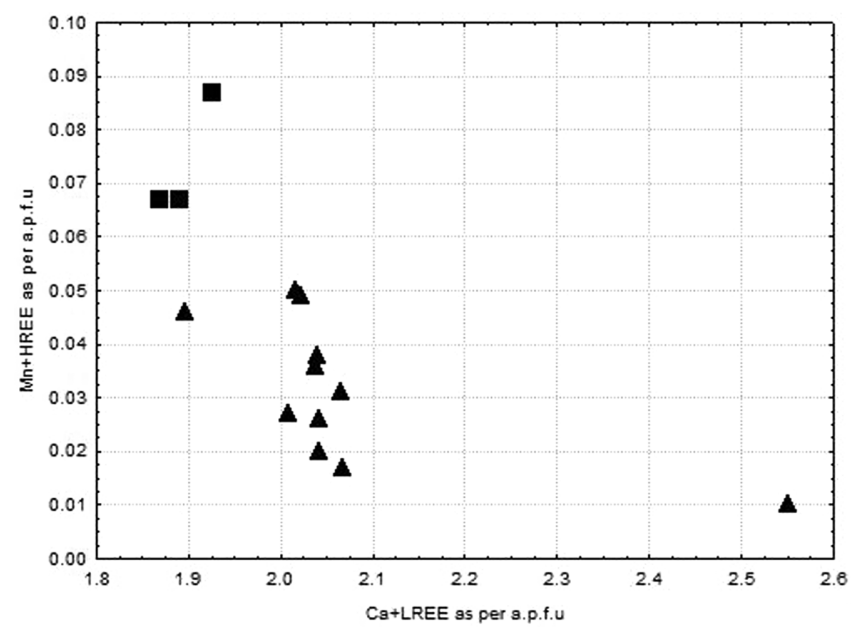

Figure 16. Plot of Mn+HREE vs Ca+LREE of allanite analysis. Filled triangle of sample PCS 1-8 (Amet Granite) and filled square of sample 20-8 (syenite/alkali feldspar syenite).

Halden, op.cit.); and also leaching out of silica from the system with concomitant enrichment of REE. The REE bearing fluid impregnated the migmatite gneiss along and across foliation planes and micro

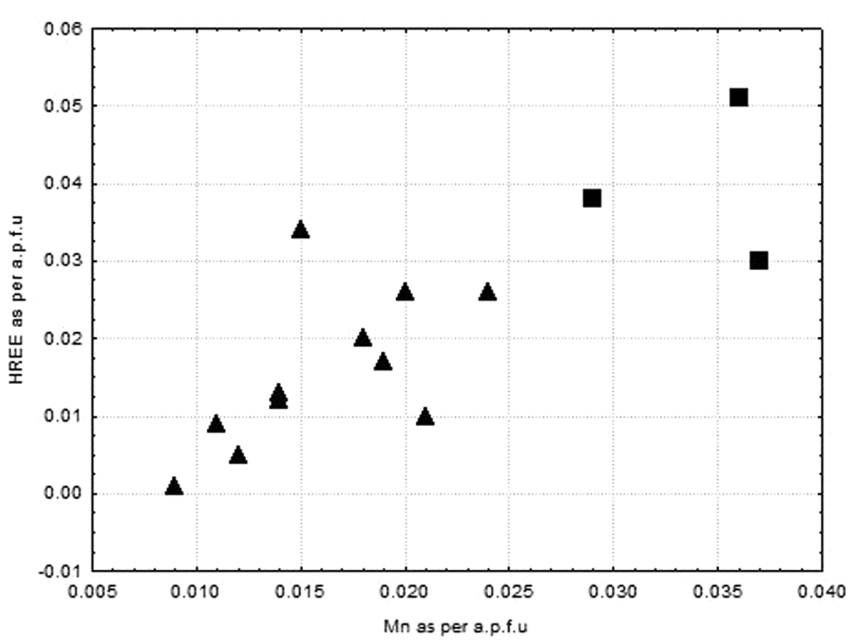

Figure 17. Plot of Mn versus HREE of allanite analysis. Filled triangle of sample PCS 1-8 (Amet Granite) and filled square of sample 20-8 (syenite/alkali feldspar syenite).

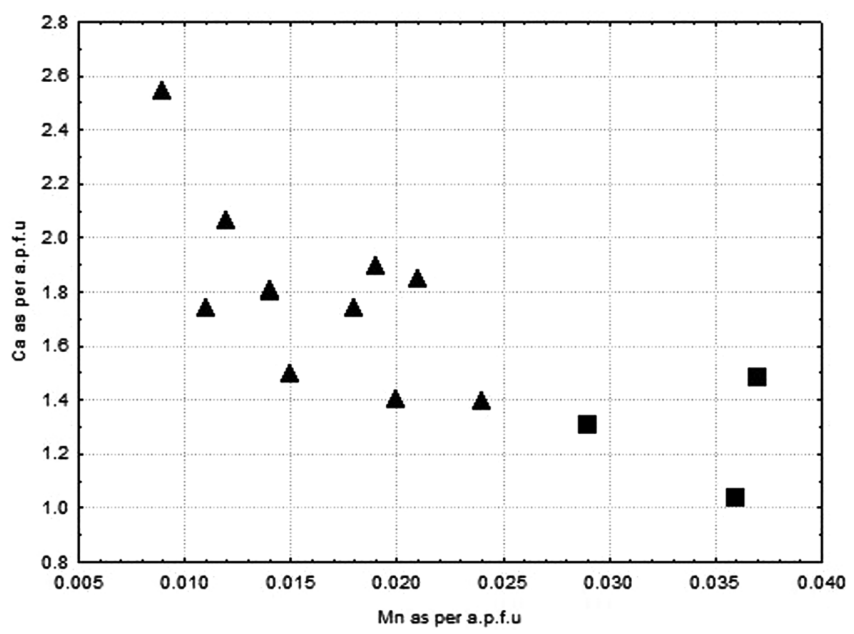

Figure 18. Plot of Mn versus Ca of allanite analysis. Filled triangle of sample PCS 1-8 (Amet Granite) and filled square of sample 20-8 (syenite/alkali feldspar syenite).

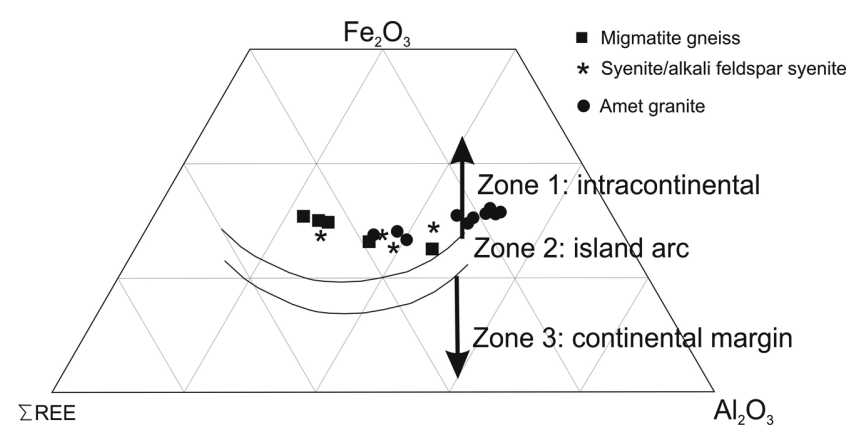

Figure 19. $\mathrm{Al}_{2} \mathrm{O}_{3}-\mathrm{Fe}_{2} \mathrm{O}_{3}-\mathrm{LREE}$ plot of allanite of migmatite gneiss, syenite-alkali feldspar syenite and Amet granite of studied area. The curved lines indicate the three groups of different kinds of occurrence of allanite (after Hoshino et al., 2005).

shears after the migmatite was crystallised. These REE bearing minerals were deposited in a conducive environment within the migmatite gneiss and Amet granite because of their (the hosts) suitable bulk 


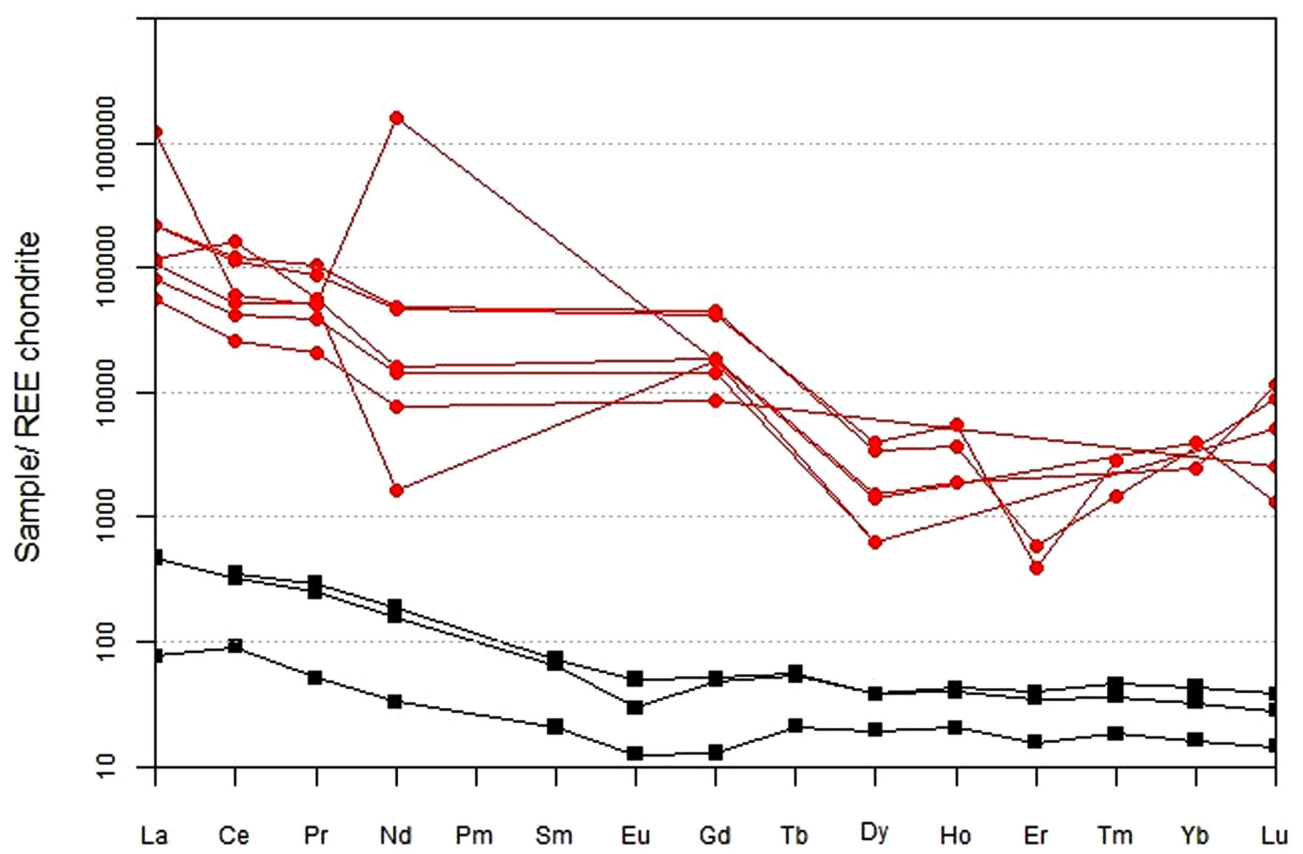

Figure 20. REE pattern of allanite versus migmatite gneiss. Red circles are allanite; Black squares are migmatite.

composition. All the cracks observed surrounding allanite grains in migmatite are expansion cracks due to change from allanite to britholite and metamictisation is not observed. This is unique in its genesis as because such large quantity of REE minerals allanite-britholitethorite-zircon-titanite-apatite is not reported from intrusive syenitealkali feldspar syenite and migmatite gneiss of the basement rocks of Rajasthan, having a large aerial extension. This report is, thus, important from REE mineral target prospecting point of view also for the Meso to Neo Archaean basement rocks of Rajasthan; and this may trigger similar finds elsewhere in India and in the world in a similar tectonic and petrological set up.

\section{Conclusions}

Allanite-britholite REE bearing migmatite gneisses, Amet Granite with its intrusive syenite-alkali feldspar syenite of the cratonic terrain of Rajasthan, India, is reported primum fama, having recoverable deposit of REE minerals. Allanite being an important REE mineral; in view of present worldwide demands of REE, this new find of REE bearing minerals in migmatite gneiss in recoverable resources will be a new emerging domain in India leading to search for REE minerals in similar terrain of India and elsewhere in the world. Concentration and the composition of REE in allanite of present finding and its host rocks; its comparison with the composition of allanite commonly occurring worldwide, established a direct relation between REE bearing allanitebritholite and its host migmatitic gneiss and other rocks as discussed above. It was established by chemico-mineralogical details of Allanite and petrochemical study of its host rocks that allanite originated from the deep-seated lower crustal intracontinental magmatic rocks syenitealkali feldspar syenite, which were the carriers of REE minerals; deposited their REE content within the host migmatite gneiss and the Amet granite along foliations, micro shears and grain boundaries in a possible peritectic condition.

In view of the large scale demands of REE, this huge quantity of recoverable REE bearing allanite from migmatitic gneiss and granite host rocks may prove to be a sumptuous deposit of REE minerals in India and similar terrains of the world.

\section{Acknowledgements}

The authors are thankful to the HOD GSI WR Jaipur for permitting to publish this paper. We are grateful to Dr. M. Mohanty Ex DDG, RSAS GSI, Bengaluru for quickly permitting to analyse the samples through EPMA at NCEGR, GSI, Bengaluru. Sri Rajendra Sharma, Ex-Director (Chem.), Sri Parvjeet Singh, Senior Chemist and team of analysts of GSI WR are thanked for XRF and ICPMS analysis of rock samples. Authors thank critical review by unknown reviewer which improved text tremendously.

\section{References}

Arden, K.M., and Halden, N.M., 1999, Crystallisation and alteration history of britholite in REE enriched pegmatitic segregations associated with the Eden Lake Complex, Manitoba Canada. The Canadian Mineralogist, v. 37, pp. 1239-1253.

Barker, F., and Arth, J.G., 1976, Generation of trondhjemitic-tonalitic liquids and Archaean bimodal trondhjemite basalt suites. Geology, v. 4, pp. 596-600.

Bose, R.M., 1989, A quantitative chemical classification of the Igneous Rocks. Indian Minerals, v. 43, pp. 47-55.

Buick, I.S., Allen, C., Pandit, M., Rubatio, D., and Hermann, J., 2006, The Proterozoic magmatic and metamorphic history of the Banded Gneissic Complex, Central Rajasthan, India: LA-ICP-MS U-Pb zircon constraints. Precambrian Research, v. 151, pp. 119-142.

Charmichael, I.S.E., Tumer, F.J., and Verhoogen, J., 1974, Igneous Petrol- 
ogy Mc.Graw-Hill, New York, pp. 27-39.

Chakrabarti, C., Pyne, T.K., Gupta, P., Basu Mallick, S., and Guha, D., 2004, A manual of the geology of India; volume I, Precambrian; Part I, southern part of the Peninsula. GSI Spl. Pub. no. 77, 257 p.

Chaudhary, A.K., Gopalan, K., and Sastry, C.A., 1984, Present status of the geochronology of the Precambrian rocks of Rajasthan. Tectonophysics, v. 105, pp. 131-140.

Condie, K.C., 1981. Archaean Greenstone Belts. Elsevier, Amsterdam, 434p.

Cox, K.G., Bell, J.D., and Pankhurst, R.J., 1979, The interpretation of igneous rocks. Boston, George Allen and Unwin London.

Dey, B., Das, K., Dasgupta, N., Bose, S., Hidaka, H., and Ghatak, H., 2018, Zircon U-Pb (SHRIMP) Ages of the Jahazpur Granite and Mangalwar Gneiss from the Deoli-Jahazpur Sector, Rajasthan, NW India: A Preliminary Reappraisal of Stratigraphic Correlation and Implications to Crustal Growth. In: Geological Evolution of the Precambrian Indian Shield, Ed. MEA Mondal. Springer, SES Series, chapter 25, pp. 39-56. doi:10.1007/978-3-319-89698-4 3.

Dharma Rao, C.V., Santosh, M., Purohit, R., Wang, J., Jiang, X., and Kusky, T., 2011, LA-ICP-MS U-Pb zircon age constraints on the Paleoproterozoic and Neoarchean history of the Sandmata Complex in Rajasthan within the NW Indian Plate. Journal of Asian Earth Sciences, v. 42, pp. 286-305.

Fareeduddin, Venkatesh, B.R., Hanumantha, R., Golani, P.R., Sharma, B. B., and Neogi, S., 2014, Petrology and stable isotope (S, C, O) studies of selected sediment-hosted basemetal ore deposits in the Proterozoic Aravalli-Delhi fold belt, Rajasthan. Journal of Geology Society India, v. 83, pp. 119-141.

Gandhi, S.M., 2003, An evaluation of the tectonic framework of the Rampura-Agucha Zinc-lead deposit.

Gopalan, K., Mc Dougall, J.D., Roy, A.B., and Murali, A.V., 1990, Sm$\mathrm{Nd}$ evidence for $3.3 \mathrm{Ga}$ old rocks in Rajasthan, northwestern India. Precambrian Research, v. 48, pp. 287-297.

Guha, D.B., 2018, Metamorphism, Crustal Evolution and Amalgamation of Meso to Neo Archaean Greenstone-Granite Craton and GranuliteGneiss Terrane in Rajasthan. In: Geological Evolution of the Precambrian Indian Shield, Ed. MEA Mondal. Springer, SES Series, chapter 25, pp. 651-693. doi:10.1007/978-3-319-89698-4 25.

Guha, D.B., and Bhattacharya, A.K., 1995, Metamorphic evolution and high grade reworking of the Sandmata Complex granulites. Memoirs of the Geological Survey of India, v. 31, pp. 163-198.

Guha, D.B., Neogi, S., and Raza, A., 2018, Evolution of a Granite GneissMigmatite Terrane in Rajasthan: Melt Generation and Origin of Anjana Granite. Geological Evolution of the Precambrian Indian Shield, Ed. MEA Mondal. Springer, SES Series, chapter 19, pp. 473-514. doi: 10.1007/978-3-319-89698-4_19.

Gupta, B.C., 1934, The geology of central Mewar. Memoir, Geological Survey of India, v. 65, pp. 107-168.

Gupta, S.N., Arora, Y.K., Mathur, R.K., Iqballuddin, Prasad, B., Sahai, T.N., and Sharma, S.B., 1997, The Precambrian geology of the Aravalli region, southern Rajasthan and north eastern Gujarat. Memoirs of the Geological Survey of India, v. 123, pp. 262.

Harmer, R.E., and Nex, P.A.M., 2016, Rare earth deposits of Africa. Episodes, v. 39, pp. 381-406.

Heron, A.M., 1953, The geology of central Rajputana. Memoirs of the Geological Survey of India, v. 79, pp. 1-389.

Hoshino, M., Kimata, M., Nishida, N., Kyono, A., Shimizu, M., and Takizawa, S., 2005, The chemistry of allanite from the Daibosatsu Pass, Yamanashi, Japan. Mineralogical Magazine, v. 69, pp. 403-423.

Irvine, T.N., and Baragar, W.R.A., 1971, A guide to the chemical classification of the common volcanic rocks. Canadian Journal of Earth Sciences, v. 8, pp. 523-548.

Kumar, S., 2014, Magmatic processes: review of some concepts and models. Modelling of magmatic and allied processes. Ed. S. Kumar and R. N. Singh. Springer, SES Series, pp. 1-22. doi: 10.1007/978-3-319-
06471-0 1.

Merlet, C., 1992, Quantitative electron probe microanalysis: New accurate $\varphi(\rho z)$ description. Microchimica Acta Supplement, v. 12, pp. 107115 .

Merlet, C., 1994, An Accurate Computer Correction Program for Quantitative Electron Probe Microanalysis. Microchim. Acta, v. 114/115, pp. 363-376.

Middlemost, E.A.K., 1985, Magmas and magmatic rocks: an introduction to igneous petrology. 266 p., London, New York.

Mohanty, M. and Guha, D.B., 1995, Lithotectonic stratigraphy of the dismembered greenstone sequence of the Mangalwar Complex around Lawa-Sardargarh and Parasali areas, Rajsamand district, Rajasthan. Memoir, Geological Society India, v. 31, pp. 141-162.

Molloy, Martin, W., 1959, A comparative study of ten monazites. American Mineralogist, v. 4, pp. 510-532.

Monrad, J.R., 1983, Evolution of sialic terrains in the vicinity of the Holenarsipur belt. Hassan district, Karnataka, India. In : Naqvi, S. M. and Rogers, J.J W. (eds.), Precambrian of South India, Memoir, Geological Society India, v. 4, pp. 343-364.

Mueller, R.F., and Saxena, S.K., 1977, Chemical Petrology: with applications to the Terrestrial Planets and Meteorites. Springer, New York, $394 \mathrm{p}$.

Nanda, L.K., Katti, V.J., and Maithani, P.B., 2009, Report, URAM, IAEA, $\mathrm{CN}-175 / 26$.

Neogi, S., Guha, D.B., and Garkhal, P., 2017, Ecandrewsite and Zincian Manganoan Ilmenite from albitite veins of South Khetri Fold Belt, Rajasthan-implications in mineral search for basemetal-gold-uranium. Episodes, v. 40, pp. 315-324.

Olson, Jerry Chipman and William N. Sharp, 1951, Geologic setting of the Mountain Pass bastnaesite deposits, San Bernardino County, California: abstract. Geological Society of America Bulletin, v. 62, 1467 p.

Ozha, M.K., Pal, D.C., Mishra, B., Desapati, T., and Shaji, T.S., 2017, Geochemistry and chemical dating of uraninite in the Samarkiya area, central Rajasthan, northwestern India Implication for geochemical and temporal evolution of uranium mineralization. Ore Geology Reviews, v. 88, pp. 23-42.

Prasad, B., 1984, Geology, Sedimentation and Palaeogeography of the Vindhyan Supergroup, southeastern Rajasthan. Memoirs of the Geological Survey of India, v. 116, p. 117.

Raja Rao, C.S., 1970, Sequence, structure and correlation of the metasediments and gneisses of the BGC of Rajasthan. Records of the Geological survey of India, v. 98, pp. 60-79.

Raja Rao, C.S., Poddar, B.C., Basu, K.K., and Dutta, A.K. 1971, Precambrian stratigraphy of Rajasthan: A review. Records of the Geological survey of India, v. 101, pp. 60-79.

Sarkar, G., Barman, T.R., and Corfu, F., 1989, Timing of continental arctype magmatism in northwest India: evidence from $\mathrm{U}-\mathrm{Pb}$ zircon geochronology. The Journal of Geology, v. 97, pp. 607-612.

Shaji, T.S., Nautiyal, K., Yadav, G.S., Yadav, O.P., Nanda, L.K., and Maithani, P.B., 2011, https://amd.gov.in/app16/content.aspx?link=689

Shand, S.J. 1943, The Eruptive Rocks. 2nd edition, John Wiley, New York, $444 \mathrm{p}$.

Sinha Roy, S., 2000, Precambrian terrane evolution in Rajasthan. Special publication, Records of the Geological survey of India, v. 55, pp. 275286.

Smith, C.A., 1982, The tectonic setting and chemical classification of the granites of the eastern marginal zone of the Mamraquakud Metamorphic Complex. Trans. South African Journal of Geology, v. 85, pp. $141-153$.

Sun, S.S., and Nesbitt, R,W., 1978, Petrogenesis of Archaean ultrabasin and basic volcanics; evidence from rare earth elements. Contributions to Mineralogy and Petrology, v. 65, pp. 301-325.

Wiedenbeck, M., Goswami, J.N., and Roy, A.B., 1996, Stabilization of Aravalli craton of the north-western India at $2.5 \mathrm{Ma}$ : An ion microprobe zircon study. Chemical Geology, v. 129, pp. 325-340.

Winkler, H.G.F., 1974, Petrogenesis of metamorphic rocks. Third Ed., 
Springer Verlag, New York. 320p.

Woyski, Margaret, S., 1980, Petrology of the Mountain Pass carbonatite complex - A review, in Geology and mineral wealth of the California Desert, Fife, D.L. and Brown A.R., Editors, South Coast Geological Society.

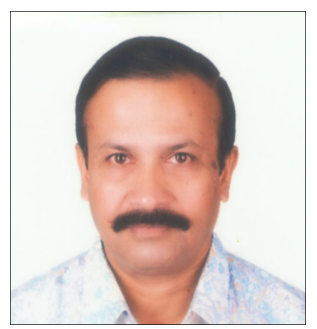

Dipendu Bhushan Guha is presently located at Jaipur, Rajasthan (India) as Deputy Director General (Retired) of Geological Survey of India. Dr. Guha obtained his Graduation (Honours) and Post-Graduation in Geology from Banaras Hindu University, Varanasi, India and did Doctorate research in the Lesser Himalayan metamorphics of Kashmir (India) from Panjab University, Chandigarh (India). He extensively studied the high grade granulites and greenstone cratonic terrane of Rajasthan, India. He established sizeable gold deposit in Rajasthan, India. He has to his credit more than forty original research papers. He has been awarded with National Mineral Award-2005 by Government of India, B. P. Radhakrishna Prize of Geological Society of India, and Geological Mining \& Metallurgical Society's silver medal for his geological findings.

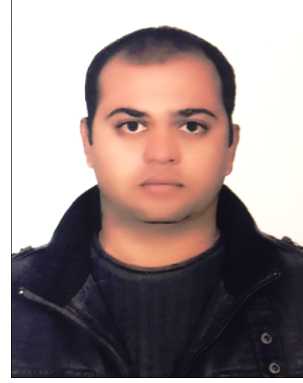

Ausaf Raza is presently located at Jaipur, Rajasthan (India) as Geologist of Geological Survey of India. Dr. Raza obtained his Graduation (Honours) and Post-Graduation in Geology from Aligarh Muslim University, Aligarh, India and did Doctorate research in Greenstone belt of the Bundelkhand craton from Aligarh Muslim University, Aligarh (India). He studied the high grade granulites of Rajasthan and greenstone cratonic terrane of Rajasthan and Bundelkhand, India. He has to his credit more than four original research papers.
Yadav, G. S., Muthamilselvan, A., Shaji, T.S., Nanda, L.K., and Rai, A.K., 2015, Recognition of a new albitite zone in northern Rajasthan: its implications on uranium mineralization. Current Science, v. 108, pp. 1994-1998.

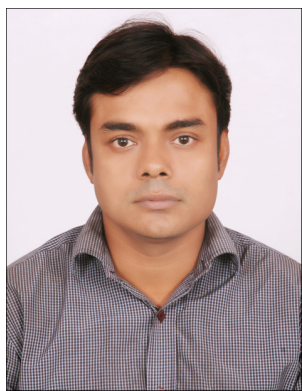

Sandip Neogi is a Senior Geologist of Geological Survey Of India, Govt Of India Ministry of Mines and presently posted in technical coordination division of State Unit- West Bengal and Andaman Nicobar at Kolkata, West Bengal (India). He did his MSc in Apllied Geology form Presidency College, Kolkata and after pursuing MSc degree he worked as a Junior Research Fellow (1.5 year) in a Department of Science and Technology, Govt Of India sponsered research project related to the evolution of Tista Dome in Sikkim- Darjeelig Himalaya in the same Institute. He has published several paper in various scientific journals and his area of interest and researh are structura geology, petrology and geochemistry.

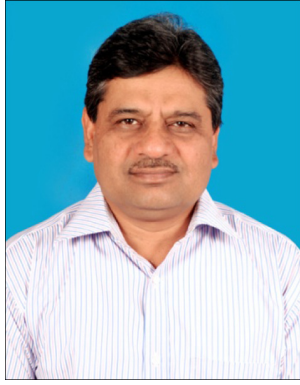

Madiwalappa Korakoppa is presently located at EPMA Lab, NCEGR, GSI, Bangalore, India as Senior Geologist of Geological Survey of India. Mr. Korakoppa obtained his Graduation, Post-Graduation, and Master of Philosophy from Karnataka University Dharwad, India. He is presently engaged in Doctorate research on Undraldoddi Kimberlite, Raichur Kimberlite Field, Karnataka at IIT (Indian School of Mines), Dhanbad, India. He has studied the greenstone belts of Dharwar Craton and Raichur Kimberlite Field, India. He has to his credit more than seventeen publications of original research papers. He has more than 10 years of experience in geological applications of EPMA instrument and provided international standard quality EPMA data to the GSI scientific officers and outside scien tific agencies and Universities of India. 